\title{
Engineering cofactor and transport mechanisms in Saccharomyces cerevisiae for enhanced acetyl-CoA and polyketide biosynthesis
}

\author{
Javier Cardenas $^{\mathrm{a}}$ and Nancy A. Da Silva ${ }^{\mathrm{a}^{*}}$ \\ ${ }^{a}$ Department of Chemical Engineering and Materials Science, University of California, Irvine, \\ CA, 92697-2575 USA \\ *Corresponding author
}

Tel: (+1) 949-824-8288

Fax: (+1) 949-824-2541

E-mail: ndasilva@uci.edu

(C) 2016. This manuscript version is made available under the Elsevier user license

http://www.elsevier.com/open-access/userlicense/1.0/ 


\begin{abstract}
Synthesis of polyketides at high titer and yield is important for producing pharmaceuticals and biorenewable chemical precursors. In this work, we engineered cofactor and transport pathways in Saccharomyces cerevisiae to increase acetyl-CoA, an important polyketide building block. The highly regulated yeast pyruvate dehydrogenase bypass pathway was supplemented by overexpressing a modified Escherichia coli pyruvate dehydrogenase complex (PDHm) that accepts $\mathrm{NADP}^{+}$for acetyl-CoA production. After $24 \mathrm{~h}$ of cultivation, a 3.7-fold increase in $\mathrm{NADPH} / \mathrm{NADP}^{+}$ratio was observed relative to the base strain, and a 2.2 -fold increase relative to introduction of the native E. coli $\mathrm{PDH}$. Both E. coli pathways increased acetyl-CoA levels approximately 2-fold relative to the yeast base strain. Combining PDHm with a ZWF1 deletion to block the major yeast NADPH biosynthesis pathway resulted in a 12-fold NADPH boost and a 2.2-fold increase in acetyl-CoA. At 48 h, only this coupled approach showed increased acetyl-CoA levels, 3.0-fold higher than that of the base strain. The impact on polyketide synthesis was evaluated in a S. cerevisiae strain expressing the Gerbera hybrida 2pyrone synthase (2-PS) for the production of the polyketide triacetic acid lactone (TAL). Titers of TAL relative to the base strain improved only $30 \%$ with the native E. coli PDH, but 3.0-fold with PDHm and 4.4-fold with PDHm in the $\Delta z w f 1$ strain. Carbon was further routed toward TAL production by reducing mitochondrial transport of pyruvate and acetyl-CoA; deletions in genes POR2, MPC2, PDA1, or YAT2 each increased titer 2 to 3 -fold over the base strain (up to 0.8 $\mathrm{g} / \mathrm{L}$ ), and in combination to $1.4 \mathrm{~g} / \mathrm{L}$. Combining the two approaches (NADPH-generating acetylCoA pathway plus reduced metabolite flux into the mitochondria) resulted in a final TAL titer of $1.6 \mathrm{~g} / \mathrm{L}$, a 6 -fold increase over the non-engineered yeast strain, and 35\% of theoretical yield $(0.16$ $\mathrm{g} / \mathrm{g}$ glucose), the highest reported to date. These biological driving forces present new avenues for improving high-yield production of acetyl-CoA derived compounds.
\end{abstract}

Key Words: Saccharomyces cerevisiae; pyruvate dehydrogenase; acetyl-CoA; mitochondrial pyruvate carrier; triacetic acid lactone 


\section{Introduction}

Polyketides are a diverse group of natural products with valuable properties useful for the manufacture of biologics and pharmaceuticals as well as replacement of fossil-fuel derived materials (Jeandet et al., 2013; Li and Vederas, 2009; Nikolau et al., 2008). The synthesis of these molecules require polyketide synthase (PKS) systems that catalyze a complex set of reactions including decarboxylation, condensation, cyclization, and aromatization (Hertweck, 2009; Jez et al., 2002). Limitations in the native host include biosynthesis of small quantities and a lack of genetic tools for strain manipulations (Jeandet et al., 2012; Lussier et al., 2012; Santos et al., 2011); therefore, engineering of heterologous microbial cell factories is of great interest (Krivoruchko and Nielsen, 2015). The yeast Saccharomyces cerevisiae is advantageous as it is capable of synthesizing fungal and plant polyketides that are toxic to or difficult to produce in bacteria (Cardenas and Da Silva, 2014; Ma et al., 2009), and also allow the synthesis of complex polyketides relying on cytochrome P450 activities (Howat et al., 2014). Various types of PKSs are active in yeast, and the synthesis of fungal polyketides (6-methylsalicylic acid, dihydromonacolin L) as biologics (Kealey et al., 1998; Ma et al., 2009; Wattanachaisaereekul et al., 2007; Xu et al., 2013) and a plant polyketide (triacetic acid lactone, TAL) as a biorenewable chemical precursor (Cardenas and Da Silva, 2014; Saunders et al., 2015; Schwartz et al., 2014) has demonstrated the promise of yeast for polyketide biosynthesis.

Many polyketides are built from the simple building blocks acetyl-CoA and malonylCoA. These starter metabolites are in high demand in yeast, e.g., for fatty acid synthesis (Jong et al., 2014) and energy via ATP (Pronk et al., 1996). In order to synthesize polyketides at high levels, it is therefore necessary to improve availability of these starter metabolites and relevant cofactor pools. One of the most direct methods for improving synthesis of acetyl-CoA and 
malonyl-CoA is by overexpressing the enzymes responsible for their formation and eliminating competing pathways. Using various overexpression and pathway disruption strategies, several studies have reported interventions aimed at increasing acetyl-CoA for the synthesis of sesquiterpenes (Chen et al., 2010, 2013; Shiba et al., 2007), butanol (Krivoruchko et al., 2013; Lian et al., 2014), polyhydroxybutyrate (PHB) (Kocharin et al., 2013, 2012), as well as fatty acids (Li et al., 2014; X. Tang et al., 2013). For malonyl-CoA formation, acetyl-CoA carboxylase (Acc1) is important and can limit malonyl-CoA synthesis. This can be circumvented by overexpression (Runguphan and Keasling, 2014; Wattanachaisaereekul et al., 2008) or prevention of deactivation of the Acc1 enzyme following glucose consumption (Choi and $\mathrm{Da}$ Silva, 2014; Hofbauer et al., 2014; Shi et al., 2014). In this recent work in our lab, the overexpression of a deactivation-resistant Acc1 yielded a 9-fold increase in activity in vivo leading to a 3-fold increase in both fatty acid and polyketide levels.

To engineer S. cerevisiae for increases in the starter metabolites acetyl-CoA and malonylCoA in the cytosol, it is also important to focus on the availability of pyruvate in the cell. Pyruvate is a major branch point for carbon following glycolysis (Pronk et al., 1996), one of which is the synthesis of acetyl-CoA via the PDH bypass (see Figure 1). Pyruvate is first decarboxylated; the Ald6 dehydrogenase then generates acetate that is converted to acetyl-CoA by the acetyl-CoA synthetase (Acs1/2). Recent computational optimizations rerouted carbon flux from the PDH bypass through an E. coli PDH to increase levels of the polyketide TAL; this also conserves ATP by eliminating use of the ATP-dependent Acs1/2 (Chowdhury et al., 2014). In this in silico study, the native PDH bypass was downregulated, and only use of an engineered $\mathrm{NADP}^{+}$-dependent $E$. coli $\mathrm{PDH}$ predicted an improved yield of TAL. Using the native $E$. coli $\mathrm{PDH}$ created a redox imbalance due to the $\mathrm{NAD}^{+}$-dependent nature of the enzyme, resulting in 
no increase in TAL yield. A recent experimental approach in $S$. cerevisiae utilized various acetyl-CoA producing pathways including the E. coli $\mathrm{PDH}$ which increased isobutanol levels; the authors commented that a reduced energy input may have been a contributing factor (Lian et al., 2014). The use of cofactors to further increase carbon flux through engineered pathways has been demonstrated for the production of diverse products in yeast, including fatty acid ethyl esters (FAEEs), polyhydroxybutyrate (PHB), and 7-dehydrocholesterol (Jong et al., 2014; Kocharin et al., 2013; Su et al., 2015). These studies support altering host cofactor levels for improved product formation.

Pyruvate can also be utilized by other pathways after its carboxylation or transport to the mitochondria. The yeast mitochondrial pyruvate carrier (MPC) has been shown to transport pyruvate into the mitochondria (Bricker et al., 2012; Herzig et al., 2012). Similarly, acetyl-CoA can be transported using the carnitine shuttle system where carnitine acetyltransferases (CATs) are responsible for first priming acetyl-CoA for transport (via Yat2) before facilitating movement into the mitochondria (Yat1) where acetyl-CoA is regenerated (Cat2) (Franken et al., 2008). Pyruvate and acetyl-CoA are thus invaluable to the cell, and rely on these systems for transport into the mitochondria causing a significant drain on their cytosolic availability. Impeding this transport may result in larger cytosolic pools and thus increase polyketide synthesis in yeast.

The overall aim of our work was to engineer novel modifications to cofactor and transport pathways in S. cerevisiae to directly increase acetyl-CoA levels and thus polyketide synthesis. To increase acetyl-CoA, we expressed (for the first time) the $\mathrm{NADP}^{+}$variant of the $E$. coli pyruvate dehydrogenase complex $(\mathrm{PDHm})$ in yeast, and $\mathrm{NADPH} / \mathrm{NADP}^{+}$ratios and acetylCoA were compared to those for the base strain and one expressing the native E. coli PDH. PDHm overexpression was then coupled with a $Z W F 1$ gene deletion (to eliminate the major 
yeast NADPH-producing pathway) to further drive carbon flux to acetyl-CoA, and the effects on the synthesis of the polyketide triacetic acid lactone (TAL) were determined. Additional pathway design incorporated key gene disruptions that reduced mitochondrial transport of pyruvate and acetyl-CoA. This novel strategy to increase cytosolic levels of pyruvate and acetyl-CoA in $S$. cerevisiae was assessed by evaluating the effects on TAL biosynthesis. The hindered transport and NADPH/acetyl-CoA upregulation strategies were then combined to further increase TAL production in the host. We demonstrate how increasing metabolite levels via these strategies can increase precursor levels and thus production of polyketides.

\section{Materials and Methods}

\subsection{Strains and plasmids}

Escherichia coli strain XL1-Blue (Stratagene, Santa Clara, CA) was used for plasmid amplification and E. coli NEB 10-beta cells (New England Biolabs, Ipswich, MA) were used for amplification of Gibson-assembled vectors. Saccharomyces cerevisiae strain BY4741 and various single gene deletion strains were obtained from Open Biosystems (Huntsville, AL). Further deletion strains were constructed using loxP-flanked MET15, LEU2-d8, TRP1, and HIS3 selection markers amplified from our pXP vector library (Fang et al., 2011). The amplification primers contained approximately 50bp homology to locations flanking the target gene location. Yeast cells were transformed as described previously (Gietz et al., 1992; Hill et al., 1991) and gene disruptions were verified following amplification of genomic DNA using Taq polymerase (New England Biolabs, Ipswich, MA). Details of the strain construction and all primer sequences can be found in the Supplementary Data (Table S1). The complete list of S. cerevisiae strains is given in Table 1. 
Plasmid pXP842-2PS harbors the G. hybrida g2ps1 gene encoding 2-pyrone synthase (Cardenas and Da Silva, 2014). To express the E. coli PDH complex, a $2 \mu$-based trigenic vector was constructed. DNA fragments were created to flank the three PDH genes with promoters and terminators as listed: ADH2p-lpd1-ADH2t, ADH1Mp-aceF-ADH2t, and HXT7p-aceE-CYCt. The three genes were isolated by PCR from E. coli chromosomal DNA preparations (Dale and Greenaway, 1985). The $l p d l$ and $a c e F$ fragments were placed in opposite orientation to prevent recombination of the terminator sequences. $A D H 1 M p$ refers to the medium truncated $A D H 1$ promoter which alters glucose-induced expression to approximately constitutive expression (Ruohonen et al., 1995) Template vector pXP843 (Leber and Da Silva, 2013) was linearized by BamHI / AatII (New England Biolabs, Ipswich, MA). Gibson assembly (Gibson et al., 2009) using the Phusion Hot Start II DNA Polymerase (New England Biolabs, Ipswich, MA) was performed using this linearized backbone and the three gene fragments listed above to generate pJCT-PDH. For construction of the alternate trigenic vector pJCT-PDHm for expression of the variant $E$. coli $\mathrm{PDHm}$ complex, the wildtype sequence for the $l p d l$ gene was replaced with a variant incorporating 15 base changes using insertion mutagenesis (Lee et al., 2010). These modifications coincide with the amino acid changes previously identified for $\mathrm{NADP}^{+}$binding (Bocanegra et al., 1993) as well as optimal codon usage preferences in S. cerevisiae (Table S2). Further details for construction of pJCT-PDH and pJCT-PDHm including all primers can be found in the Supplementary Data (Table S1, Figure S1). All plasmids used and constructed can be found in Table 1. Unless otherwise stated, all PCR-based amplifications were performed using KOD Hot Start DNA Polymerase (EMD Chemicals, San Diego, CA). Oligonucleotides used in this study were provided by IDT DNA (San Diego, CA), the GeneJet ${ }^{\text {TM }}$ Plasmid Miniprep Kit (Thermo Scientific, Waltham, MA) was used for plasmid preparation, and DNA 
sequences were confirmed for all PCR-amplified inserts (Eton Biosciences, San Diego, CA).

\subsection{Media and cultivation}

Luria-Bertani (LB) media was used for cultivation of XL1-Blue cells, with $150 \mathrm{mg} / \mathrm{L}$ ampicillin added for selection of plasmid-containing cells (Sambrook and Russell, 2001). Yeast selective SDC medium ( $2 \%$ dextrose, $0.5 \%$ casamino acids, $0.67 \%$ yeast nitrogen base, $0.5 \%$ ammonium sulfate supplemented with $100 \mathrm{mg} / \mathrm{L}$ adenine sulfate) and SD minimal medium (2\% dextrose, $0.67 \%$ yeast nitrogen base, $0.5 \%$ ammonium sulfate and supplemented with $100 \mathrm{mg} / \mathrm{L}$ adenine sulfate, $100 \mathrm{mg} / \mathrm{L}$ L-tryptophan, $100 \mathrm{mg} / \mathrm{L}$ L-histidine-HCL, $100 \mathrm{mg} / \mathrm{L}$ L-methionine, and/or $150 \mathrm{mg} / \mathrm{L}$ L-leucine) were used for seed cultures. Complex YPD medium (1\% dextrose, $1 \%$ yeast extract, $2 \%$ peptone) was used for the NADPH/NADP ${ }^{+}$and acetyl-CoA quantification studies and for TAL expression. The $g 2 p s 1, l p d l$, and $l p d l \mathrm{~m}$ genes were expressed from the $S$. cerevisiae late-phase $A D H 2$ promoter that is active after glucose depletion and has highest expression levels in $1 \%$ glucose YPD medium (Lee and Da Silva, 2005). Due to the late expression, plasmid stabilities remain high even in this complex medium (Shen et al., 2012).

S. cerevisiae strains were grown overnight in $5 \mathrm{~mL}$ selective media in an air shaker (New Brunswick Scientific) at $250 \mathrm{rpm}$ and $30^{\circ} \mathrm{C}$, and used to inoculate $5 \mathrm{~mL}$ tube cultures to an initial cell density $\left(\mathrm{OD}_{600}\right)$ of 0.3 (Shimadzu UV-2450 UV-VIS Spectrophotometer, Columbia, MD). A correlation factor was used to convert OD measurements to dry cell weight per liter (Cardenas and Da Silva, 2014). For NADPH/NADP ${ }^{+}$and acetyl-CoA quantification, samples were taken at 12, 24, and $48 \mathrm{~h}$. For measuring polyketide TAL titers in the culture broth, the supernatant at $48 \mathrm{~h}$ was collected for HPLC analysis. 


\subsection{Plasmid stability}

The yeast strains BYt and BYtB $\Delta z w f 1 \Delta p o r 2 \Delta m p c 2 \Delta p d a 1 \Delta y a t 2$ (Table 1) transformed with pXP842-2PS and pJCT-PDHm were cultivated in 1\% YPD medium for $48 \mathrm{~h}$, and plated onto YPD plates following serial dilutions with sterile water. After colonies were allowed to grow for 2-3 days, cells were replica plated to SDC plates supplemented with either $100 \mathrm{mg} / \mathrm{L} \mathrm{L-}$ tryptophan or $100 \mathrm{mg} / \mathrm{L}$ uracil, as well as to non-selective YPD plates (to verify cell viability and transfer). The percentage of plasmid-containing cells was determined as the number of colonies on the selective plates divided by the number of viable colonies transferred. The plasmid stability was calculated for each individual plasmid as well as in combination. Three independent experiments were performed.

\subsection{Quantitative NADPH / NADP ${ }^{+}$assay}

Both NADPH and $\mathrm{NADP}^{+}$were quantified using a colorimetric 96-well plate assay (Sigma-Aldrich, St Louis, MO). Following a cold buffer (PBS) wash of the cells, a combination of 2 freeze / thaw cycles and homogenization was performed prior to deproteinization using a 10 $\mathrm{kD}$ cut-off spin filter (Sigma-Aldrich, St. Louis, MO). The cycling reaction was performed, and absorbance taken at $450 \mathrm{~nm}$. A SpectraMax plate reader using SoftMax software was used for data acquisition (Molecular Devices, Sunnyvale, CA).

\subsection{Acetyl-CoA assay}

A boiling ethanol extraction was used on collected cell pellets for acetyl-CoA analysis, based on published procedures (Gonzalez et al., 1997; Villas-Bôas et al., 2005). Following release of the metabolites, nitrogen-supported solvent evaporation provided rapid sample concentration at room temperature. Following complete solvent evaporation, $200 \mu \mathrm{L}$ Sigma 
acetyl-CoA assay buffer was added for resuspension. A fluorescence-based 96-well acetyl-CoA plate assay was used (Sigma-Aldrich, St. Louis, MO), and concentrated acetyl-CoA identified using wavelengths $535 \mathrm{~nm}$ (excitation) and $587 \mathrm{~nm}$ (emission) on a SpectraMax plate reader using SoftMax software for data acquisition.

\subsection{Polyketide detection by HPLC}

The concentration of triacetic acid lactone was measured by HPLC using the following Shimadzu configuration: LC-10AT pumps (Shimadzu), UV-VIS detector (SPD-10A VP, Shimadzu), and Zorbax SB-C18 reversed-phase column (2.1x150 mm, Agilent Technologies). Acetic acid-buffered (1\%) acetonitrile and water were used as the mobile and aqueous phases, respectively. A gradient program using a 95-85\% Pump B gradient $\left(\mathrm{H}_{2} \mathrm{O}\right.$ with $1 \%$ acetic acid) provided an elution time of approximately 12 minutes (flow rate $0.25 \mathrm{~mL} / \mathrm{min}$, column temperature $\left.25^{\circ} \mathrm{C}\right)$.

\section{Results and Discussion}

\subsection{Enhancement of NADPH/NADP+ and acetyl-CoA using PDH or PDHm overexpression}

The synthesis of polyketides requires the availability of starter metabolites that react to form the final product. Triacetic acid lactone (TAL) is an acetyl-CoA derived polyketide that requires one acetyl-CoA and two malonyl-CoA units. Since cytosolic malonyl-CoA is formed directly from acetyl-CoA, the pool of acetyl-CoA was increased through a non-native pathway. The E. coli pyruvate dehydrogenase complex (PDH), responsible for converting pyruvate to acetyl-CoA, was introduced to supplement the highly-regulated and ATP-requiring yeast pathway. To further increase utilization of this heterologous pathway, a modified NADP ${ }^{+}$ utilizing E. coli PDH variant (PDHm) (Bocanegra et al., 1993) was constructed and introduced to Page | 10 
simultaneously resupply NADPH and acetyl-CoA levels (Figure 1).

The impact of the cofactor preference of PDHm and PDH was first characterized by measuring the NADPH/NADP ${ }^{+}$ratios for base strain $\mathrm{BYt}+\mathrm{pXP} 843$ (carrying an empty vector), strain BYt+pJCT-PDH, and strain BYt+pJCT-PDHm during batch cultivation. The ratios measured at 24 and $48 \mathrm{~h}$ are shown in Figure 2. Formation of active PDH complex was limited by expression of the lpdl gene from the $S$. cerevisiae late-phase $A D H 2$ promoter that is only active after glucose depletion ( $\mathrm{t}>12 \mathrm{~h}$ ). At $12 \mathrm{~h}$, the three strains all had similar ratios (data not shown). However, at $24 \mathrm{~h}$, the strain expressing the modified PDH (PDHm) had an increased cofactor ratio, with $\mathrm{NADPH} / \mathrm{NADP}^{+}$ratio 3.7 -fold greater than the base strain and 2.2-fold greater than the strain expressing the native E. coli PDH. The modified PDH thus increased NADPH availability in the cell. By $48 \mathrm{~h}$, the strains had similar ratios again, indicating routine utilization of NADPH as a reducing agent during stationary phase. Observing these differences well after glucose is depleted and during ethanol utilization indicates the PDH complex has a sufficient supply of pyruvate. This could be traced to respiratory metabolism and ethanol breakdown to acetate following the diauxic shift (Galdieri et al., 2010). As acetate is further assimilated to acetyl-CoA in both the cytosol and mitochondria, respiratory function commits acetyl-CoA to both the glyoxylate and TCA cycles where oxaloacetate formation becomes important (Chen et al., 2012; Haurie et al., 2001). Pyruvate can then be replenished during ethanol-grown conditions by one of two paths: direct synthesis from phosphoenolpyruvate formed from oxaloacetate, or direct synthesis from malate which is an intermediate metabolite of the glyoxylate and TCA cycles (Boles et al., 1998). This well-characterized carbon flow is also consistent with our observation that high NADPH levels are present in our engineered system, as NADPH regulates glyoxylate function under these conditions (Satrustegui et al., 1983; Ying, 
2008).

Expression of the E. coli PDH complex may also improve acetyl-CoA levels as this pathway supplements the native yeast pyruvate dehydrogenase bypass pathway. With in vivo PDH activity indirectly verified by the improved cofactor ratios, the levels of acetyl-CoA were then measured for base strain BYt+pXP843 (empty vector), strain BYt+pJCT-PDH, and strain BYt+pJCT-PDHm during batch culture (Figure 3). At $24 \mathrm{~h}$, the strains expressing PDH and PDHm showed 2.3-fold and 2.4-fold increases in acetyl-CoA, respectively, similar to observations at $12 \mathrm{~h}$ (data not shown). By $48 \mathrm{~h}$ the PDH overexpression system (pJCT-PDH, Figure 3) had a 58\% reduction in acetyl-CoA levels relative to the base strain. This may be attributed to the $\mathrm{NAD}^{+}$consumption associated with the native E. coli complex and corresponding cofactor imbalance (Chowdhury et al., 2014). Recent computational studies demonstrated the importance of cofactor preference when introducing the PDH complex in $S$. cerevisiae for TAL synthesis (Chowdhury et al., 2014), and our experimental results corroborate this requirement. While PDH overexpression can improve acetyl-CoA levels, introducing a resupply of reducing equivalents via the NADPH-generating variant (PDHm) is more beneficial, resulting in increases in both the NADPH/NADP ${ }^{+}$ratio and acetyl-CoA levels.

\subsection{NADPH driving force further increases cofactor and acetyl-CoA pools}

Introduction of the NADPH-generating $\mathrm{PDH}$ variant $(\mathrm{PDHm})$ alone was capable of increasing flux toward acetyl-CoA. Elimination of the pentose phosphate pathway (the major NADPH-generating pathway in yeast) via deletion of ZWF1 (encoding glucose-6-phosphate dehydrogenase) should further improve utilization of this new route for acetyl-CoA, as the new pathway will now be a major source of NADPH for the cell (Figure 1).

Therefore, NADPH/NADP ${ }^{+}$and acetyl-CoA levels were measured for ZWF1 knockout Page | 12 
strains BYt $\Delta z w f 1+\mathrm{pXP} 843$ (empty vector) and BYt $\Delta z w f 1+\mathrm{pJCT}-\mathrm{PDHm}$, and compared with the strains with an intact ZWF1 (Figures 2 and 3). Deletion of ZWF1 had limited effect on the $\mathrm{NADPH} / \mathrm{NADP}+$ ratio. This was not surprising due to the various known alternate routes for generating NADPH in S. cerevisiae (Grabowska and Chelstowska, 2003). However, the coupled strain $(\Delta z w f 1+$ PDHm expression) had a 2.9 -fold higher cofactor ratio level than the base strain at $12 \mathrm{~h}$ (data not shown). By $24 \mathrm{~h}$, the NADPH/NADP ${ }^{+}$ratio for the coupled strain was over $10-$ fold that of the base strain (Figure 2). Another notable outcome of this coupled strain was the observed change in acetyl-CoA levels (Figure 3). All strains with PDH overexpression (pJCTPDH, pJCT-PDHm, and $\Delta z w f 1+$ pJCT-PDHm) exhibited over 2.2 to 2.4 -fold increases in acetylCoA after $24 \mathrm{~h}$ relative to the base strain. However, coupled strain BYt $\Delta z w f 1+\mathrm{pJCT}$-PDHm was the only one that maintained high acetyl-CoA levels at $48 \mathrm{~h}$. This indicates that a large change in yeast cofactor and acetyl-CoA levels takes place only when a driving force (knockout of the

pentose phosphate pathway) has been implemented. The increased levels of NADPH and acetylCoA can be applied toward the biosynthesis of polyketides or other acetyl-CoA derived molecules.

\subsection{Improved polyketide levels using PDHm in the cofactor-deficient strain}

The overexpression of PDHm in the $\Delta z w f l$ strain increased both NADPH and acetyl-CoA availability, with acetyl-CoA levels higher at late stages of the cultivation. To determine the effect on polyketide synthesis, we transformed these previously tested strains with pXP842-2PS, carrying the G. hybrida g2ps1 (encoding 2-pyrone synthase; 2-PS) under the late-phase $A D H 2$ promoter. TAL levels were measured after $48 \mathrm{~h}$ of batch cultivation (Figure 4). Co-expression of 2-PS with the native E. coli $\mathrm{PDH}$ generated $0.32 \mathrm{~g} / \mathrm{L}$ TAL, a $30 \%$ increase relative to the base yeast strain. This was the smallest increase of all strains tested, indicating that the cofactor 
imbalance introduced with the $\mathrm{NAD}^{+}$-consuming PDH may limit the possible improvement. Coexpression of 2-PS with the modified E. coli $\mathrm{PDH}(\mathrm{PDHm})$, resulted in a 3-fold increase in titer to $0.75 \mathrm{~g} / \mathrm{L}$ TAL. To create a driving force for acetyl-CoA, the ZWF1 deletion blocking the major NADPH-generating pathway was important (Figure 3), and should lead to increased TAL production. This $\Delta z w f 1$ modification alone was previously shown to result in TAL increases, as pentose biosynthesis is a significant carbon drain from glycolysis (Cardenas and Da Silva, 2014). In the current study, knocking out $Z W F 1$ alone resulted in a 2.6-fold improvement over the base strain TAL. Expression of the native E. coli $\mathrm{PDH}$ in the $\Delta z w f 1$ strain gave no significant increase in TAL levels relative to the $\Delta z w f 1$ strain alone. However, coupling the $\Delta z w f 1$ strain with PDHm overexpression increased TAL levels an additional $70 \%$ relative to BYt $\Delta z w f 1$, and 4.5 -fold higher relative to the base yeast strain. This supports the computational modeling by Chowdhury et al. (2014). Therefore, introduction of this biological driving force for acetyl-CoA production via PDHm improved the acetyl-CoA pools (Figure 3), and thus increased TAL synthesis (Figure 4) in late batch culture. This strategy may prove even more useful for polyketides that utilize NADPH for their synthesis, and where an even greater NADPH pool is required.

\subsection{Eliminating mitochondrial precursor transport pathways}

Our overall aim was to direct carbon toward cytosolic acetyl-CoA for polyketide production. In S. cerevisiae, various shuttling events involving acetyl-CoA and pyruvate can limit the availability of this metabolite. Therefore, we considered two main routes, the mitochondrial pyruvate carrier (MPC) and the carnitine shuttle system, as well as the common mitochondrial porins (Figure 5). For the MPC, gene products Mpc1, Mpc2, and Mpc3 form $\mathrm{Mpc} 1 / \mathrm{Mpc} 2$ and $\mathrm{Mpc} 1 / \mathrm{Mpc} 3$ complexes (Mpc2 and Mpc3 are paralogs), part of the active transport mechanism (Herzig et al., 2012). Using the MPC, pyruvate transport into the 
mitochondrial matrix and subsequent conversion by decarboxylation commits this carbon flow toward mitochondrial acetyl-CoA (Figure 5). Replenishing pyruvate will be important to effectively use our engineered PDHm platform. Similarly, cytosolic acetyl-CoA can be transported to the mitochondria via the carnitine shuttle system (Franken et al., 2008) instead of being utilized in the cytosol (Figure 5). Therefore, limiting transport of pyruvate and acetyl-CoA from the cytoplasm while minimizing deleterious effects on the cell may be an effective means of increasing polyketide production.

We first identified a group of eight proteins involved in pyruvate and acetyl-CoA transport and conversion (Figure 5), and evaluated the effects of their single gene knockouts on both growth and TAL production. Seven of the proteins are in pathways that facilitate transport of various metabolites across the outer membrane by porins (Por1, Por2), specific transport of pyruvate from the intermembrane space into the mitochondrial matrix (Mpc1, Mpc2, Mpc3), and transport of acetyl-CoA from the cytosol into the intermembrane space (Yat1, Yat2). The knockout strains were transformed with pXP842-2PS and samples were taken at $48 \mathrm{~h}$. Por1 and Por2 are responsible for the mitochondrial permeability of charged solutes including pyruvate, ATP, and phosphate by forming voltage-dependent, anion-selective channels (VDACs) in the outer membrane (Blachly-Dyson et al., 1997). Due to differences in their activity, the $\Delta$ porl and $\triangle p o r 2$ strains produced very different levels of TAL (Figure 6). While $\triangle p o r 2$ increased TAL 2.5fold to $0.62 \mathrm{~g} / \mathrm{L}, \Delta$ porl nearly abolished product formation with a $94 \%$ reduction in titer relative to the parent strain. This is consistent with reports that Por1 maintains mitochondrial osmotic stability (Galganska et al., 2008). Por2 is not associated with this activity, and instead represses Sod1 (superoxide dismutase) that in turn alleviates respiration repression. Additionally, $\Delta$ por 1 has been found to suffer a growth defect due to reduced respiratory function on non-fermentable 
carbon, in contrast to the increase under the same conditions for $\Delta$ por2 (Dihanich et al., 1987; Sánchez et al., 2001). This is consistent with the growth observed for the culture; the $\Delta$ porl strain reached a final density equal to one-third (4.2 g DCW/L) that of the base strain (Figure 6).

The disruption of each subunit in the mitochondrial pyruvate carrier ( $\mathrm{Mpc} 1, \mathrm{Mpc} 2$, Mpc3) also resulted in different polyketide levels. The $\Delta m p c 1$ strain showed no significant effect on TAL titer relative to the base strain, while $\Delta m p c 2$ and $\Delta m p c 3$ strains provided 3.4 -fold and 1.9-fold increases in TAL titer, respectively. Final cell densities were $86 \%, 89 \%$, and $74 \%$ of the base strain for $\Delta m p c 1, \Delta m p c 2$, and $\Delta m p c 3$, respectively. The $\mathrm{Mpc} 1 \mathrm{p} / \mathrm{Mpc} 3 \mathrm{p}$ complex is necessary for retaining strong respiration and its expression is closely tied to stress responses (Timón-Gómez et al., 2013), consistent with the data showing the lower cell density for the $\Delta m p c 3$ strain. The data therefore indicates an intact $\mathrm{Mpc} 1 / \mathrm{Mpc} 3$ pyruvate carrier and disruption of the Mpc1/Mpc2 complex supports respiratory function while likely increasing cytosolic pyruvate levels leading to the significant improvement in TAL synthesis. The ability to convert pyruvate to acetyl-CoA in the mitochondria is eliminated by deleting PDAl (Figure 5); this deletion results in no detectable pyruvate decarboxylase activity (Gey et al., 2008). The BYt $\Delta p d a 1$ strain produced $>2$-fold more TAL than the base yeast strain $(0.58 \mathrm{~g} / \mathrm{L})$. This indicates that eliminating this pathway can improve titers of acetyl-CoA-derived compounds like TAL.

For cytosolic acetyl-CoA transport to the mitochondria, the first two committed steps from the carnitine shuttle system were evaluated. Yat1 and Yat2 are responsible for committing acetyl-CoA to the carnitine shuttle system and transporting it to the mitochondrial matrix, respectively (Franken et al., 2008; Swiegers et al., 2001) (Figure 5). In the $\Delta y a t 1$ strain, a 94\% drop in TAL was observed, while in the $\Delta y a t 2$ strain, TAL levels increased over 2-fold relative 
to the base strain (Figure 6). The data indicates that the cell is much more tolerant of eliminating the cytosolic Yat2 than the mitochondrial Yat1; the mitochondrial Yat1 is responsible for shuttling acetyl-CoA from other compartments like the peroxisome and may be more critical for proper cell function. In addition, Yat2 is highly active when ethanol is used as the carbon source (Franken et al., 2008; Swiegers et al., 2001), the same time that TAL synthesis is occurring with the $A D H 2$ promoter, and thus deleting this function may be advantageous. We did not consider a CAT2 deletion strain (Figure 5) as Cat2 has activity in both the mitochondria and the peroxisome (Franken et al., 2008).

\subsection{Combining transport gene disruptions with engineered NADPH driving force}

From the eight genes tested for disruption, four were chosen for combination to further reduce the flow of pyruvate and acetyl-CoA transport into the mitochondria. With the single knockout strains, significant improvements in TAL levels were observed by disrupting $\Delta$ por2, $\Delta m p c 2, \Delta p d a 1$, and $\Delta y a t 2$. Therefore, we deleted all four genes in a single strain and measured TAL levels (Figure 7, Panels A-C). Cultivation of strain BYt $\Delta$ por2 yielded $0.62 \mathrm{~g} / \mathrm{L}$ TAL with a reduction in biomass of $10 \%$ relative to the base strain. Knockout of MPC2 further elevated titers to $0.98 \mathrm{~g} / \mathrm{L}$ TAL, while also reducing biomass by an additional $20 \%$. Further interventions had no negative effect on biomass, and incorporation of $\Delta p d a 1$ and $\Delta y a t 2$ led to additional titer increases to 1.27 and $1.33 \mathrm{~g} / \mathrm{L}$, corresponding to a final yield on glucose of $0.13 \mathrm{~g} / \mathrm{g}$ ( $28 \%$ of theoretical yield). The diminishing improvements observed with the sequential knockouts suggest that other pathways are now limiting the production of TAL. This strain was then transformed with pJCT-PDHm to determine if coupling PDHm expression with the transport knockouts would further increase TAL levels. However, no significant increase was observed.

To also introduce a NADPH driving force, the four deletions were incorporated in a Page | 17 
$\Delta z w f 1$ strain. TAL levels were measured after $48 \mathrm{~h}$ of batch culture (Figure 7, Panels D-F). Plasmid stability did not change significantly even in this highly engineered strain. For both BYt and BYtB $\Delta z w f 1 \Delta p o r 2 \Delta m p c 2 \Delta p d a 1 \Delta y a t 2,61-67 \%$ of the cells retained both plasmids after 48 hours of growth (Table S3). As shown previously (Figure 4), deletion of ZWF1 alone led to increased TAL levels, likely due to the improved carbon flux through glycolysis. Similar increases were seen for the strains with the sequential transport knockouts; a 2-fold improvement was achieved by incorporating this four-gene disruption ( $\Delta$ por $2 \Delta m p c 2 \Delta p d a 1 \Delta y a t 2)$ into the $\Delta z w f l$ strain. However, in this case, subsequent transformation with pJCT-PDHm led to a further significant increase in specific TAL production and in titer $(\mathrm{p}<0.001)$. The combination of approaches led to a final titer of $1.6 \mathrm{~g} / \mathrm{L}$ TAL, corresponding to $35 \%$ of the theoretical yield on glucose $(0.16 \mathrm{~g} / \mathrm{g})$, the highest reported value for batch fermentation (Figure 8). These novel strategies thus proved effective in maintaining high pyruvate and acetyl-CoA levels in the cytosol, which was in turn used for polyketide formation. 


\section{Conclusions}

S. cerevisiae has been shown to be amenable to significant metabolic rewiring for producing acetyl-CoA derived compounds. In this study, we created a biological driving force that could increase acetyl-CoA pools applicable toward producing polyketides. Characterization of the modified NADPH-generating E. coli $\mathrm{PDH}$ (PDHm) relative to the native E. coli $\mathrm{PDH}$ indicated that NADPH synthesis was beneficial even though TAL biosynthesis does not require it directly. Coupling this with a $\Delta z w f 1$ strain (to create additional driving force for acetyl-CoA synthesis) led to TAL levels 4.4-fold higher than the parent strain. This improvement was attributed to increased NADPH/NADP ${ }^{+}$and acetyl-CoA levels, up to 10-fold and 3-fold higher than in the parent. To further increase the availability of acetyl-CoA for polyketide synthesis, gene deletions were used to reduce transport of acetyl-CoA and pyruvate into the mitochondria. The single gene deletions in POR2, MPC2, PDA1, and YAT2 each provided over a 3-fold increase in titer (up to $0.84 \mathrm{~g} / \mathrm{L}$ ) relative to the parent strain. Coupling these four in a single knockout strain ( $\Delta p o r 2 \Delta m p c 2 \Delta p d a 1 \Delta y a t 2)$ provided increased titer to $1.33 \mathrm{~g} / \mathrm{L}$. Introducing this four-gene disruption into the coupled $\Delta z w f 1$ / pJCT-PDHm strain provided an additional $50 \%$ improvement in TAL titer to $1.6 \mathrm{~g} / \mathrm{L}$. This is $35 \%$ of the theoretical yield on glucose $(0.16 \mathrm{~g} / \mathrm{g})$, the highest reported value for batch cultivation. This coupled strategy illustrates a promising step toward improving strain development for synthesis of acetyl-CoA derived compounds. Additional features can also be incorporated to further enhance acetyl-CoA pools, including reduced byproduct formation (Cardenas and Da Silva, 2014), enzyme engineering (Cardenas, 2015; Tang et al., 2013), and downregulation of competing essential pathways such as fatty acid biosynthesis. 


\section{Acknowledgements}

This research was supported by the National Science Foundation (Grant No. EEC-0813570) through the Engineering Research Center CBiRC (Center for Biorenewable Chemicals). 


\section{References}

Blachly-Dyson, E., Song, J., Wolfgang, W.J., Colombini, M., Forte, M., 1997. Multicopy suppressors of phenotypes resulting from the absence of yeast VDAC encode a VDAC-like protein. Mol. Cell. Biol. 17, 5727-5738.

Bocanegra, J.A., Scrutton, N.S., Perham, R.N., 1993. Creation of an NADP-dependent pyruvate dehydrogenase multienzyme complex by protein engineering. Biochemistry 32, 2737-2740.

Boles, E., de Jong-Gubbels, P., Pronk, J.T., 1998. Identification and Characterization of MAE1, the Saccharomyces cerevisiae Structural Gene Encoding Mitochondrial Malic Enzyme. Journal of bacteriology 180, 2875-2882.

Bricker, D.K., Taylor, E.B., Schell, J.C., Orsak, T., Boutron, A., Chen, Y.-C., Cox, J.E., Cardon, C.M., Vranken, J.G.V., Dephoure, N., Redin, C., Boudina, S., Gygi, S.P., Brivet, M., Thummel, C.S., Rutter, J., 2012. A Mitochondrial Pyruvate Carrier Required for Pyruvate Uptake in Yeast, Drosophila, and Humans. Science 337, 96-100. doi:10.1126/science.1218099

Cardenas, J., 2015. Metabolic Engineering of Saccharomyces cerevisiae for the High-level Synthesis of Polyketides. University of California, Irvine.

Cardenas, J., Da Silva, N.A., 2014. Metabolic engineering of Saccharomyces cerevisiae for the production of triacetic acid lactone. Metabolic Engineering 25, 194-203. doi:10.1016/j.ymben.2014.07.008

Chen, F., Zhou, J., Shi, Z., Liu, L., Du, G., Chen, J., 2010. Effect of acetyl-CoA synthase gene overexpression on physiological function of Saccharomyces cerevisiae. Wei Sheng Wu Xue Bao $50,1172-1179$.

Chen, Y., Daviet, L., Schalk, M., Siewers, V., Nielsen, J., 2013. Establishing a platform cell factory through engineering of yeast acetyl-CoA metabolism. Metabolic Engineering 15, 48-54. doi:10.1016/j.ymben.2012.11.002

Chen, Y., Siewers, V., Nielsen, J., 2012. Profiling of Cytosolic and Peroxisomal Acetyl-CoA Metabolism in Saccharomyces cerevisiae. PLoS ONE 7, e42475. doi:10.1371/journal.pone.0042475

Choi, J.W., Da Silva, N.A., 2014. Improving polyketide and fatty acid synthesis by engineering of the yeast acetyl-CoA carboxylase. Journal of Biotechnology 187, 56-59. doi:10.1016/j.jbiotec.2014.07.430

Chowdhury, A., Zomorrodi, A.R., Maranas, C.D., 2014. k-OptForce: Integrating Kinetics with Flux Balance Analysis for Strain Design. PLoS Comput Biol 10, e1003487. doi:10.1371/journal.pcbi.1003487

Dale, J.W., Greenaway, P.J., 1985. Preparation of Chromosomal DNA from E. coli. Methods Mol. Biol. 2, 197-200. doi:10.1385/0-89603-064-4:197

Dihanich, M., Suda, K., Schatz, G., 1987. A yeast mutant lacking mitochondrial porin is respiratorydeficient, but can recover respiration with simultaneous accumulation of an 86-kd extramitochondrial protein. EMBO J 6, 723-728.

Fang, F., Salmon, K., Shen, M.W.Y., Aeling, K.A., Ito, E., Irwin, B., Tran, U.P.C., Hatfield, G.W., Da Silva, N.A., Sandmeyer, S., 2011. A vector set for systematic metabolic engineering in Saccharomyces cerevisiae. Yeast 28, 123-136. doi:10.1002/yea.1824 
Franken, J., Kroppenstedt, S., Swiegers, J.H., Bauer, F.F., 2008. Carnitine and carnitine acetyltransferases in the yeast Saccharomyces cerevisiae: a role for carnitine in stress protection. Curr Genet 53, 347-360. doi:10.1007/s00294-008-0191-0

Galdieri, L., Mehrotra, S., Yu, S., Vancura, A., 2010. Transcriptional Regulation in Yeast during Diauxic Shift and Stationary Phase. OMICS: A Journal of Integrative Biology 14, 629-638. doi:10.1089/omi.2010.0069

Galganska, H., Budzinska, M., Wojtkowska, M., Kmita, H., 2008. Redox regulation of protein expression in Saccharomyces cerevisiae mitochondria: Possible role of VDAC. Archives of Biochemistry and Biophysics 479, 39-45. doi:10.1016/j.abb.2008.08.010

Gey, U., Czupalla, C., Hoflack, B., Rödel, G., Krause-Buchholz, U., 2008. Yeast Pyruvate Dehydrogenase Complex Is Regulated by a Concerted Activity of Two Kinases and Two Phosphatases. J. Biol. Chem. 283, 9759-9767. doi:10.1074/jbc.M708779200

Gibson, D.G., Young, L., Chuang, R.-Y., Venter, J.C., Hutchison, C.A., Smith, H.O., 2009. Enzymatic assembly of DNA molecules up to several hundred kilobases. Nat Meth 6, 343-345. doi:10.1038/nmeth.1318

Gietz, D., Jean, A.S., Woods, R.A., Schiestl, R.H., 1992. Improved method for high efficiency transformation of intact yeast cells. Nucl. Acids Res. 20, 1425-1425. doi:10.1093/nar/20.6.1425

Gonzalez, B., François, J., Renaud, M., 1997. A rapid and reliable method for metabolite extraction in yeast using boiling buffered ethanol. Yeast 13, 1347-1355. doi:10.1002/(SICI)10970061(199711)13:14<1347::AID-YEA176>3.0.CO;2-O

Grabowska, D., Chelstowska, A., 2003. The ALD6 gene product is indispensable for providing NADPH in yeast cells lacking glucose-6-phosphate dehydrogenase activity. J. Biol. Chem. 278, 1398413988. doi:10.1074/jbc.M210076200

Haurie, V., Perrot, M., Mini, T., Jeno, P., Sagliocco, F., Boucherie, H., 2001. The Transcriptional Activator Cat8p Provides a Major Contribution to the Reprogramming of Carbon Metabolism during the Diauxic Shift in Saccharomyces cerevisiae. Journal of Biological Chemistry 276, 7685. doi:10.1074/jbc.M008752200

Hertweck, C., 2009. The Biosynthetic Logic of Polyketide Diversity. Angewandte Chemie International Edition 48, 4688-4716. doi:10.1002/anie.200806121

Herzig, S., Raemy, E., Montessuit, S., Veuthey, J.-L., Zamboni, N., Westermann, B., Kunji, E.R.S., Martinou, J.-C., 2012. Identification and Functional Expression of the Mitochondrial Pyruvate Carrier. Science 337, 93-96. doi:10.1126/science.1218530

Hill, J., Donald, K.A.I.G., Griffiths, D.E., 1991. DMSO-enhanced whole cell yeast transformation. Nucl. Acids Res. 19, 5791-5791. doi:10.1093/nar/19.20.5791

Hofbauer, H.F., Schopf, F.H., Schleifer, H., Knittelfelder, O.L., Pieber, B., Rechberger, G.N., Wolinski, H., Gaspar, M.L., Kappe, C.O., Stadlmann, J., Mechtler, K., Zenz, A., Lohner, K., Tehlivets, O., Henry, S.A., Kohlwein, S.D., 2014. Regulation of Gene Expression through a Transcriptional Repressor that Senses Acyl-Chain Length in Membrane Phospholipids. Developmental Cell 29, 729-739. doi:10.1016/j.devcel.2014.04.025

Howat, S., Park, B., Oh, I.S., Jin, Y.-W., Lee, E.-K., Loake, G.J., 2014. Paclitaxel: biosynthesis, production and future prospects. New Biotechnology 31, 242-245. doi:10.1016/j.nbt.2014.02.010

Jeandet, P., Delaunois, B., Aziz, A., Donnez, D., Vasserot, Y., Cordelier, S., Courot, E., 2012. Metabolic Engineering of Yeast and Plants for the Production of the Biologically Active Hydroxystilbene, Resveratrol. BioMed Research International 2012, e579089. doi:10.1155/2012/579089

Page | 22 
Jeandet, P., Vasserot, Y., Chastang, T., Courot, E., 2013. Engineering Microbial Cells for the Biosynthesis of Natural Compounds of Pharmaceutical Significance. BioMed Research International 2013, e780145. doi:10.1155/2013/780145

Jez, J.M., Bowman, M.E., Noel, J.P., 2002. Expanding the biosynthetic repertoire of plant type III polyketide synthases by altering starter molecule specificity. Proc Natl Acad Sci U S A 99, 53195324. doi:10.1073/pnas.082590499

Jong, B.W. de, Shi, S., Siewers, V., Nielsen, J., 2014. Improved production of fatty acid ethyl esters in Saccharomyces cerevisiae through up-regulation of the ethanol degradation pathway and expression of the heterologous phosphoketolase pathway. Microbial Cell Factories 13, 39. doi:10.1186/1475-2859-13-39

Kealey, J.T., Liu, L., Santi, D.V., Betlach, M.C., Barr, P.J., 1998. Production of a Polyketide Natural Product in Nonpolyketide-Producing Prokaryotic and Eukaryotic Hosts. Proceedings of the National Academy of Sciences of the United States of America 95, 505-509.

Kocharin, K., Chen, Y., Siewers, V., Nielsen, J., 2012. Engineering of acetyl-CoA metabolism for the improved production of polyhydroxybutyrate in Saccharomyces cerevisiae. AMB Express 2, 52. doi:10.1186/2191-0855-2-52

Kocharin, K., Siewers, V., Nielsen, J., 2013. Improved polyhydroxybutyrate production by Saccharomyces cerevisiae through the use of the phosphoketolase pathway. Biotechnology and Bioengineering n/a-n/a. doi:10.1002/bit.24888

Krivoruchko, A., Nielsen, J., 2015. Production of natural products through metabolic engineering of Saccharomyces cerevisiae. Current Opinion in Biotechnology 35, 7-15. doi:10.1016/j.copbio.2014.12.004

Krivoruchko, A., Serrano-Amatriain, C., Chen, Y., Siewers, V., Nielsen, J., 2013. Improving biobutanol production in engineered Saccharomyces cerevisiae by manipulation of acetyl-CoA metabolism. Journal of Industrial Microbiology \& Biotechnology 40, 1051-1056. doi:10.1007/s10295-0131296-0

Leber, C., Da Silva, N.A., 2013. Engineering of Saccharomyces cerevisiae for the synthesis of short chain fatty acids. Biotechnology and Bioengineering. doi:10.1002/bit.25021

Lee, J., Shin, M.-K., Ryu, D.-K., Kim, S., Ryu, W.-S., 2010. Insertion and deletion mutagenesis by overlap extension PCR. Methods Mol. Biol. 634, 137-146. doi:10.1007/978-1-60761-652-8_10

Lee, M.K., Da Silva, N.A., 2005. Evaluation of the Saccharomyces cerevisiae ADH2 promoter for protein synthesis. Yeast 22, 431-440. doi:10.1002/yea.1221

Lian, J., Si, T., Nair, N.U., Zhao, H., 2014. Design and construction of acetyl-CoA overproducing Saccharomyces cerevisiae strains. Metabolic Engineering 24, 139-149. doi:10.1016/j.ymben.2014.05.010

Li, J.W.-H., Vederas, J.C., 2009. Drug discovery and natural products: end of an era or an endless frontier? Science 325, 161-165.

Li, X., Guo, D., Cheng, Y., Zhu, F., Deng, Z., Liu, T., 2014. Overproduction of fatty acids in engineered Saccharomyces cerevisiae. Biotechnology and bioengineering 111, 1841-1852.

Lussier, F.-X., Colatriano, D., Wiltshire, Z., Page, J.E., Martin, V.J.J., 2012. Engineering Microbes for Plant Polyketide Biosynthesis. Computational and Structural Biotechnology Journal 3, 1-11. doi:10.5936/csbj.201210020 
Ma, S.M., Li, J.W.-H., Choi, J.W., Zhou, H., Lee, K.K.M., Moorthie, V.A., Xie, X., Kealey, J.T., Silva, N.A.D., Vederas, J.C., Tang, Y., 2009. Complete Reconstitution of a Highly Reducing Iterative Polyketide Synthase. Science 326, 589-592. doi:10.1126/science.1175602

Nikolau, B.J., Perera, M.A.D.N., Brachova, L., Shanks, B., 2008. Platform biochemicals for a biorenewable chemical industry. Plant J. 54, 536-545. doi:10.1111/j.1365-313X.2008.03484.x

Pronk, J.T., Yde Steensma, H., Van Dijken, J.P., 1996. Pyruvate Metabolism in Saccharomyces cerevisiae. Yeast 12, 1607-1633. doi:10.1002/(SICI)1097-0061(199612)12:16<1607::AIDYEA70>3.0.CO;2-4

Runguphan, W., Keasling, J.D., 2014. Metabolic engineering of Saccharomyces cerevisiae for production of fatty acid-derived biofuels and chemicals. Metabolic Engineering 21, 103-113. doi:10.1016/j.ymben.2013.07.003

Ruohonen, L., Aalto, M.K., Keränen, S., 1995. Modifications to the ADH1 promoter of Saccharomyces cerevisiae for efficient production of heterologous proteins. Journal of Biotechnology 39, 193203. doi:16/0168-1656(95)00024-K

Sambrook, J.J., Russell, D.D.W., 2001. Molecular cloning: a laboratory manual. Vol. 2. CSHL Press.

Sánchez, N.S., Pearce, D.A., Cardillo, T.S., Uribe, S., Sherman, F., 2001. Requirements of Cyc2p and the Porin, Por1p, for Ionic Stability and Mitochondrial Integrity in Saccharomyces cerevisiae. Archives of Biochemistry and Biophysics 392, 326-332. doi:10.1006/abbi.2001.2465

Santos, C.N.S., Koffas, M., Stephanopoulos, G., 2011. Optimization of a heterologous pathway for the production of flavonoids from glucose. Metabolic Engineering 13, 392-400. doi:16/j.ymben.2011.02.002

Saunders, L.P., Bowman, M.J., Mertens, J.A., Silva, N.A.D., Hector, R.E., 2015. Triacetic acid lactone production in industrial Saccharomyces yeast strains. J Ind Microbiol Biotechnol 1-11. doi:10.1007/s10295-015-1596-7

Schwartz, T.J., Johnson, R.L., Cardenas, J., Okerlund, A., Da Silva, N.A., Schmidt-Rohr, K., Dumesic, J.A., 2014. Engineering Catalyst Microenvironments for Metal-Catalyzed Hydrogenation of Biologically Derived Platform Chemicals. Angew. Chem. Int. Ed. n/a-n/a. doi:10.1002/anie.201407615

Shen, M.W.Y., Fang, F., Sandmeyer, S., Da Silva, N.A., 2012. Development and characterization of a vector set with regulated promoters for systematic metabolic engineering in Saccharomyces cerevisiae. Yeast 29, 495-503. doi:10.1002/yea.2930

Shiba, Y., Paradise, E.M., Kirby, J., Ro, D.-K., Keasling, J.D., 2007. Engineering of the pyruvate dehydrogenase bypass in Saccharomyces cerevisiae for high-level production of isoprenoids. Metabolic Engineering 9, 160-168. doi:10.1016/j.ymben.2006.10.005

Shi, S., Chen, Y., Siewers, V., Nielsen, J., 2014. Improving Production of Malonyl Coenzyme A-Derived Metabolites by Abolishing Snf1-Dependent Regulation of Acc1. mBio 5, e01130-14. doi:10.1128/mBio.01130-14

Su, W., Xiao, W.-H., Wang, Y., Liu, D., Zhou, X., Yuan, Y.-J., 2015. Alleviating Redox Imbalance Enhances 7-Dehydrocholesterol Production in Engineered Saccharomyces cerevisiae. PLOS ONE 10, e0130840. doi:10.1371/journal.pone.0130840

Swiegers, J.H., Dippenaar, N., Pretorius, I.S., Bauer, F.F., 2001. Carnitine-dependent metabolic activities in Saccharomyces cerevisiae: three carnitine acetyltransferases are essential in a carnitinedependent strain. Yeast 18, 585-595. doi:10.1002/yea.712 
Tang, S.-Y., Qian, S., Akinterinwa, O., Frei, C.S., Gredell, J.A., Cirino, P.C., 2013. Screening for Enhanced Triacetic Acid Lactone Production by Recombinant Escherichia coli Expressing a Designed Triacetic Acid Lactone Reporter. J. Am. Chem. Soc. 135, 10099-10103. doi:10.1021/ja402654z

Tang, X., Feng, H., Chen, W.N., 2013. Metabolic engineering for enhanced fatty acids synthesis in Saccharomyces cerevisiae. Metabolic Engineering 16, 95-102. doi:10.1016/j.ymben.2013.01.003

Timón-Gómez, A., Proft, M., Pascual-Ahuir, A., 2013. Differential Regulation of Mitochondrial Pyruvate Carrier Genes Modulates Respiratory Capacity and Stress Tolerance in Yeast. PLoS One 8. doi:10.1371/journal.pone.0079405

Villas-Bôas, S.G., Højer-Pedersen, J., Åkesson, M., Smedsgaard, J., Nielsen, J., 2005. Global metabolite analysis of yeast: evaluation of sample preparation methods. Yeast 22, 1155-1169. doi:10.1002/yea.1308

Wattanachaisaereekul, S., Lantz, A.E., Nielsen, M.L., Andrésson, O.S., Nielsen, J., 2007. Optimization of heterologous production of the polyketide 6-MSA in Saccharomyces cerevisiae. Biotechnology and Bioengineering 97, 893-900.

Wattanachaisaereekul, S., Lantz, A.E., Nielsen, M.L., Nielsen, J., 2008. Production of the polyketide 6MSA in yeast engineered for increased malonyl-CoA supply. Metabolic Engineering 10, 246254. doi:10.1016/j.ymben.2008.04.005

Xu, W., Chooi, Y.-H., Choi, J.W., Li, S., Vederas, J.C., Da Silva, N.A., Tang, Y., 2013. Finding the Missing Link in Lovastatin Biosynthesis: LovG is the Thioesterase Required for Dihydromonacolin L Release and Lovastatin Nonaketide Synthase Turnover. Angew Chem Int Ed Engl 52. doi:10.1002/anie.201302406

Ying, W., 2008. NAD ${ }^{+} / \mathrm{NADH}$ and NADP ${ }^{+} / \mathrm{NADPH}$ in Cellular Functions and Cell Death: Regulation and Biological Consequences. Antioxidants \& Redox Signaling 10, 179-206. doi:10.1089/ars.2007.1672 


\section{Figure Captions}

Figure 1: General engineering strategy applied for introducing biological driving forces. (A) Yeast relies on the pentose phosphate biosynthetic pathway (PP Pathway) as the major route for NADPH generation. To make acetyl-CoA in the cytoplasm, the native pyruvate dehydrogenase bypass (PDH bypass, green arrows) converts pyruvate to acetyl-CoA in three steps, requiring ATP. The acetyl-CoA can then be used for polyketide synthesis. (B) Introduction of the E. coli PDH $(E c P D H)$ enhances pyruvate conversion toward acetyl-CoA using a protein complex (red arrow). (C) Our engineered cofactor-coupled pathway blocks flux through the pentose phosphate pathway (crossed out), and replaces $E c P D H$ with the modified E. coli pyruvate dehydrogenase complex (EcPDHm, blue arrow) to produce acetyl-CoA and create a new NADPH supply.

Figure 2: NADPH/NADP ${ }^{+}$ratios for BYt strains harboring the native E. coli $\mathrm{PDH}$ (pJCT-PDH), the NADPH-generating variant PDH (pJCT-PDHm), and/or the $\Delta z w f 1$ gene knockout. Values at $24 \mathrm{~h}$ (hatched) and $48 \mathrm{~h}$ (solid) correspond to mean values \pm SEM ( $\mathrm{n}=2$ independent experiments).

Figure 3: Acetyl-CoA levels for BYt strains harboring the native E. coli PDH (pJCT-PDH), the NADPH-generating variant PDH (pJCT-PDHm), and/or the $\Delta z w f 1$ gene knockout. Values at $24 \mathrm{~h}$ (hatched) and $48 \mathrm{~h}$ (solid) correspond to mean values \pm SEM ( $\mathrm{n}=2$ independent experiments).

Figure 4: TAL titers (g/L) after 48 cultivation for BYt strains harboring the native E. coli PDH (pJCT-PDH), the NADPH-generating variant PDH (pJCT-PDHm), and/or the $\Delta z w f 1$ gene knockout. All strains carry pXP842-2PS for TAL synthesis. Values correspond to mean values \pm SEM ( $\mathrm{n}=6$ independent experiments).

Figure 5: Metabolic pathways related to the transport and utilization of pyruvate and acetyl-CoA, the starter metabolite for subsequent TAL formation (catalyzed by 2-PS). Pathways highlighted include the major native NADPH biosynthesis pathway (Zwf1), mitochondrial pyruvate carrier (MPC) system (Mpc1/Mpc2 and Mpc1/Mpc3), mitochondrial porins (Por1 and Por2), the mitochondrial yeast PDH (Lat1/Pda1/Pdb1/Pdx1), and carnitine shuttle system (Yat1, Yat2, Crc1, Cat2). Reactions chosen for disruption in this study have been crossed out. 
Figure 6: TAL titers (g/L, Panel A), specific TAL levels (g/g DCW, Panel B), and biomass (g DCW/L, Panel C) after 48 h cultivation of strains with single gene knockouts in pathways for the mitochondrial transport and utilization of pyruvate and acetyl CoA. All strains carry pXP8422PS for TAL synthesis. Bars represent mean values \pm SEM ( $n=6$ independent experiments).

Figure 7: TAL titers (g/L,), specific levels (g/g DCW), and biomass (g DCW/L) in strains carrying pJCT-PDHm engineered for transport alone ( $\Delta p o r 2 \Delta m p c 2 \Delta p d a 1 \Delta y a t 2$, Panels A-C), or in combination with $\Delta z w f 1$ (Panels D-F), after $48 \mathrm{~h}$ cultivation. All strains carry pXP842-2PS for TAL synthesis. Bars represent mean values \pm SEM ( $n=6$ independent experiments).

Figure 8: Final titer and yield summary for TAL production in engineered $S$. cerevisiae strains. Volumetric titer $(\mathrm{g} / \mathrm{L})$, specific production (cell basis; g/g DCW), and corresponding yield on glucose $(\mathrm{g} / \mathrm{g})$ with percent theoretical yield are shown. 
c) Optimal cofactor-coupled pathway

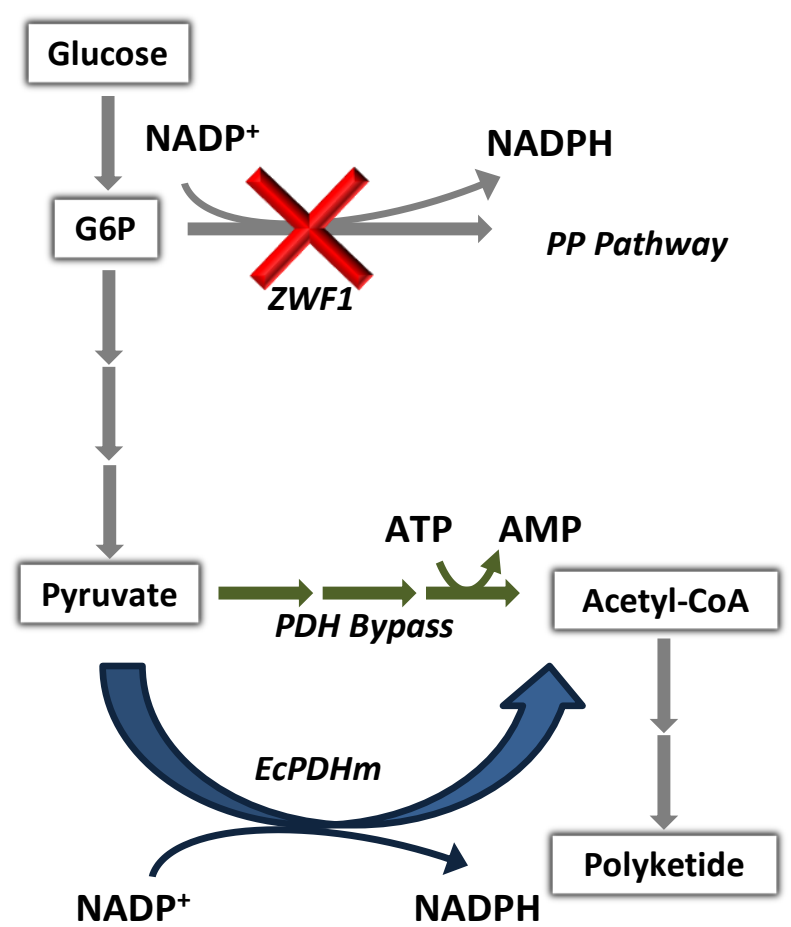

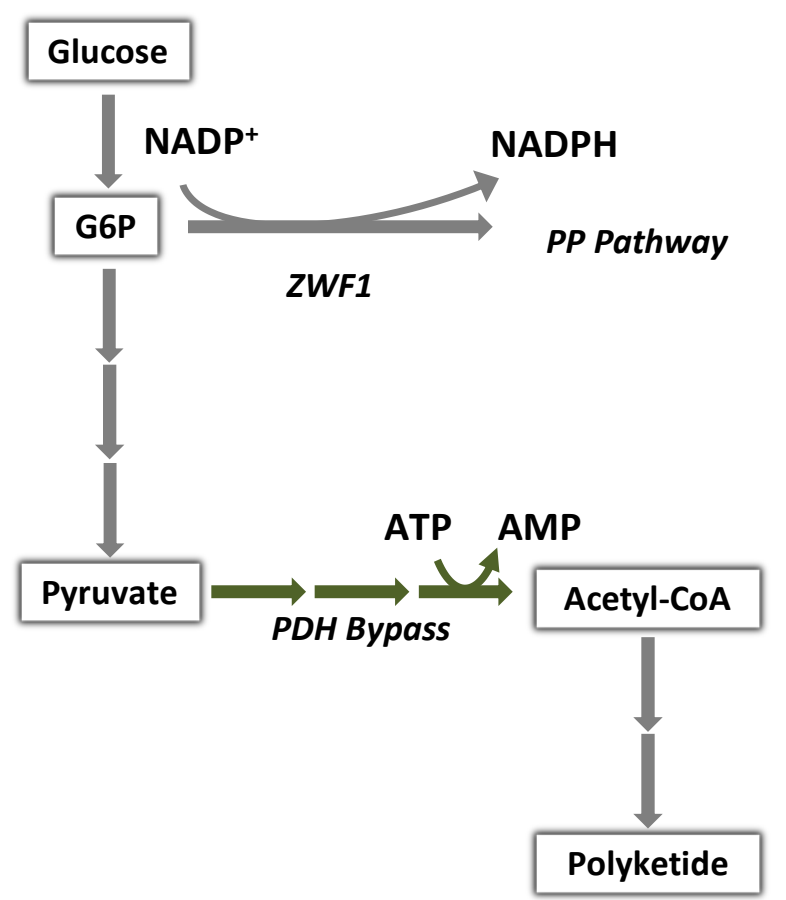

a) Native pathway

.

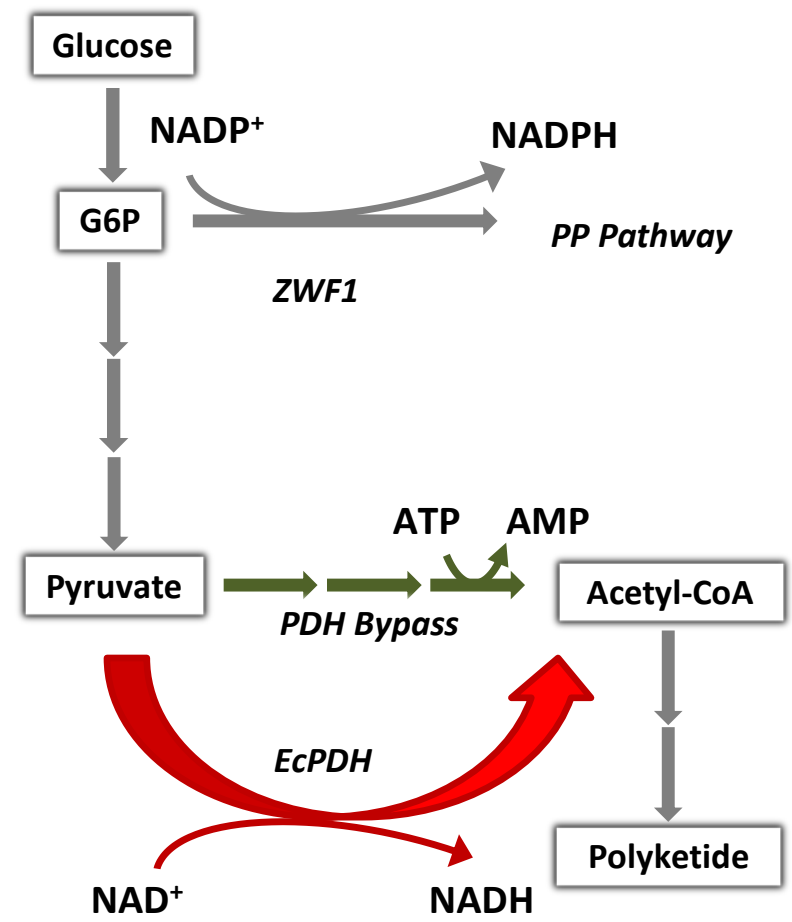

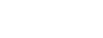




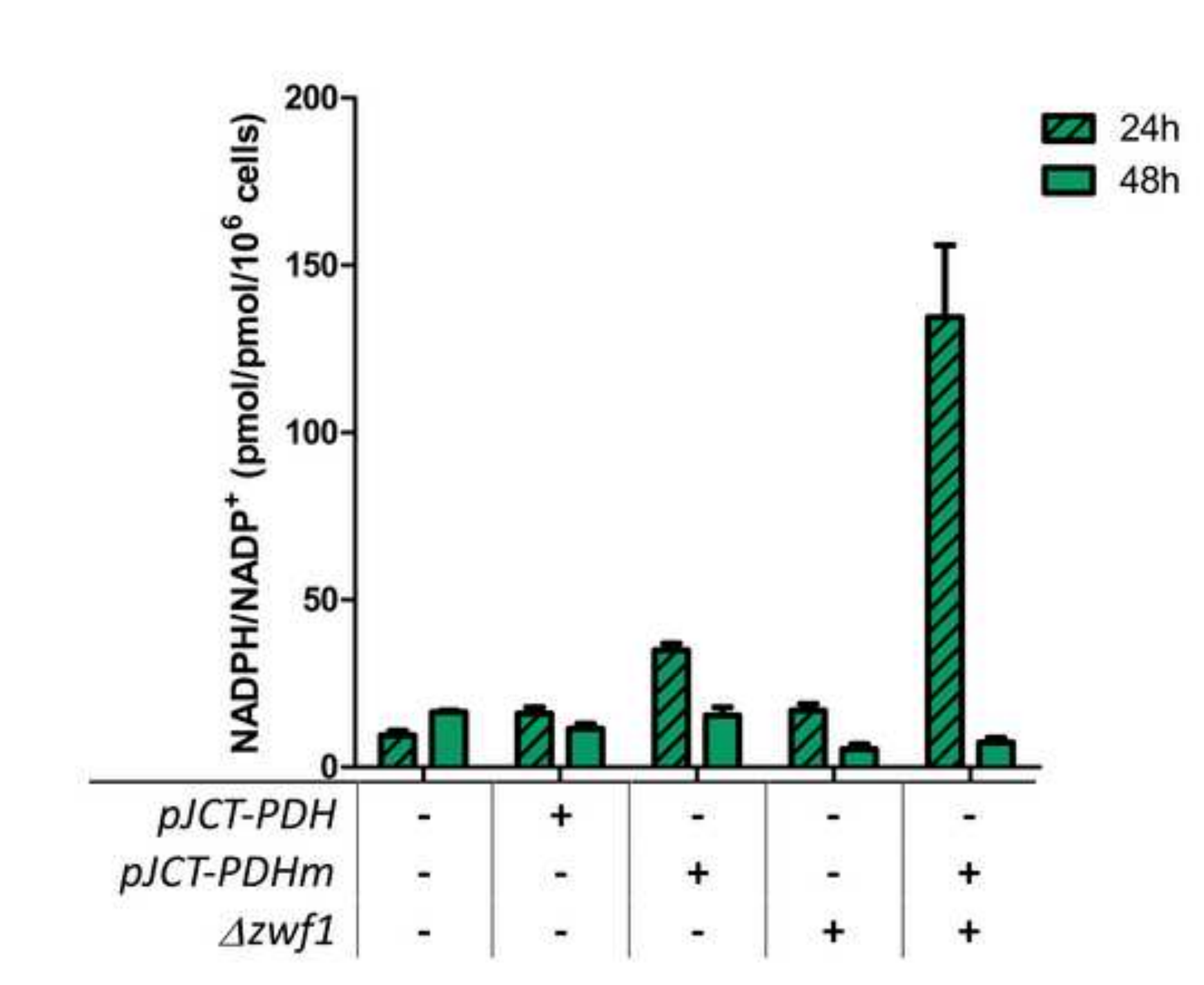

Figure 2

\section{Fure 2}

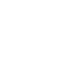




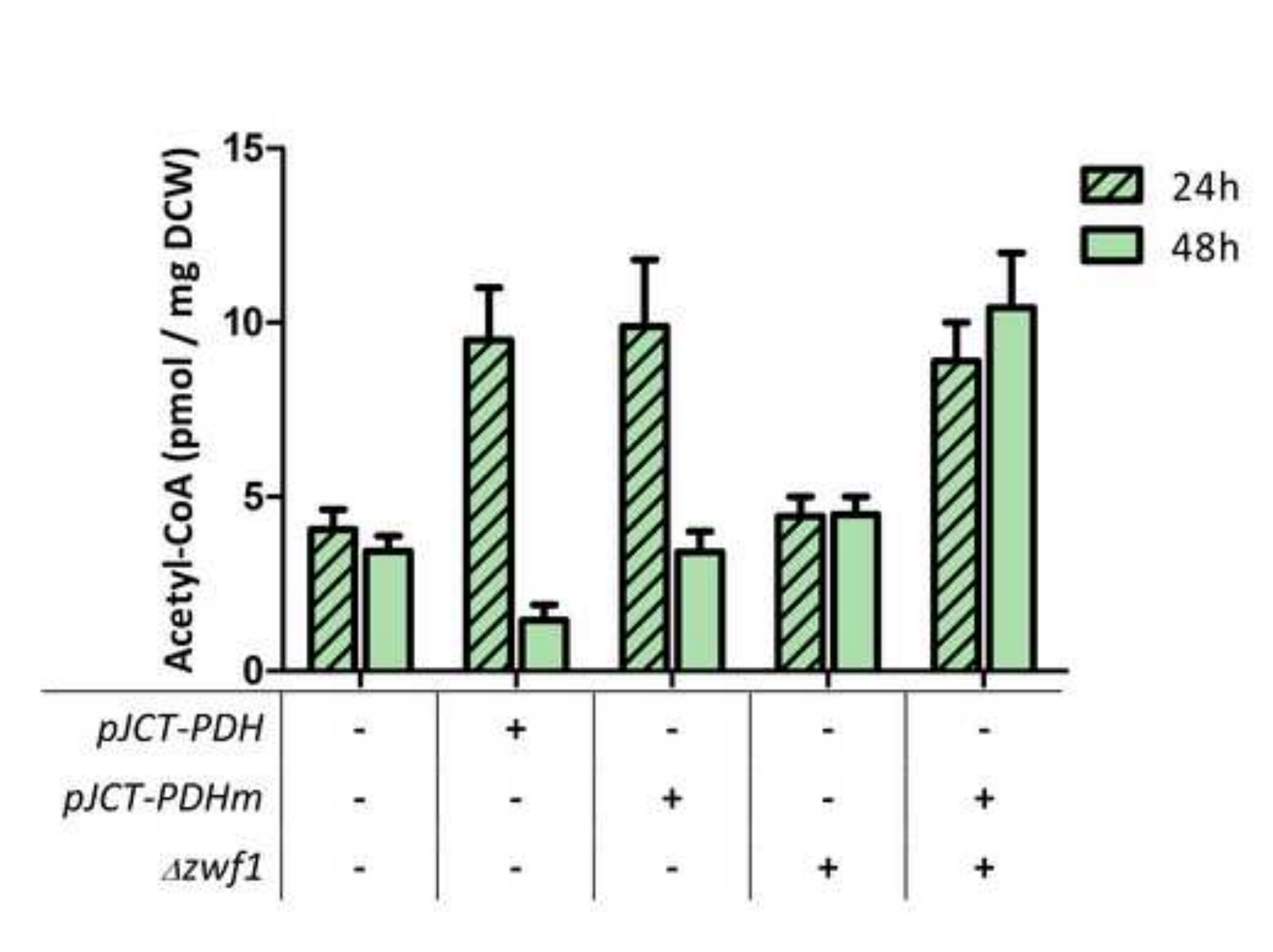

Figure 3

3

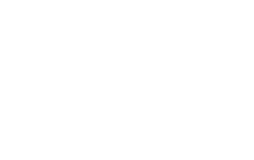

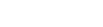

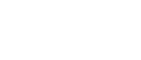
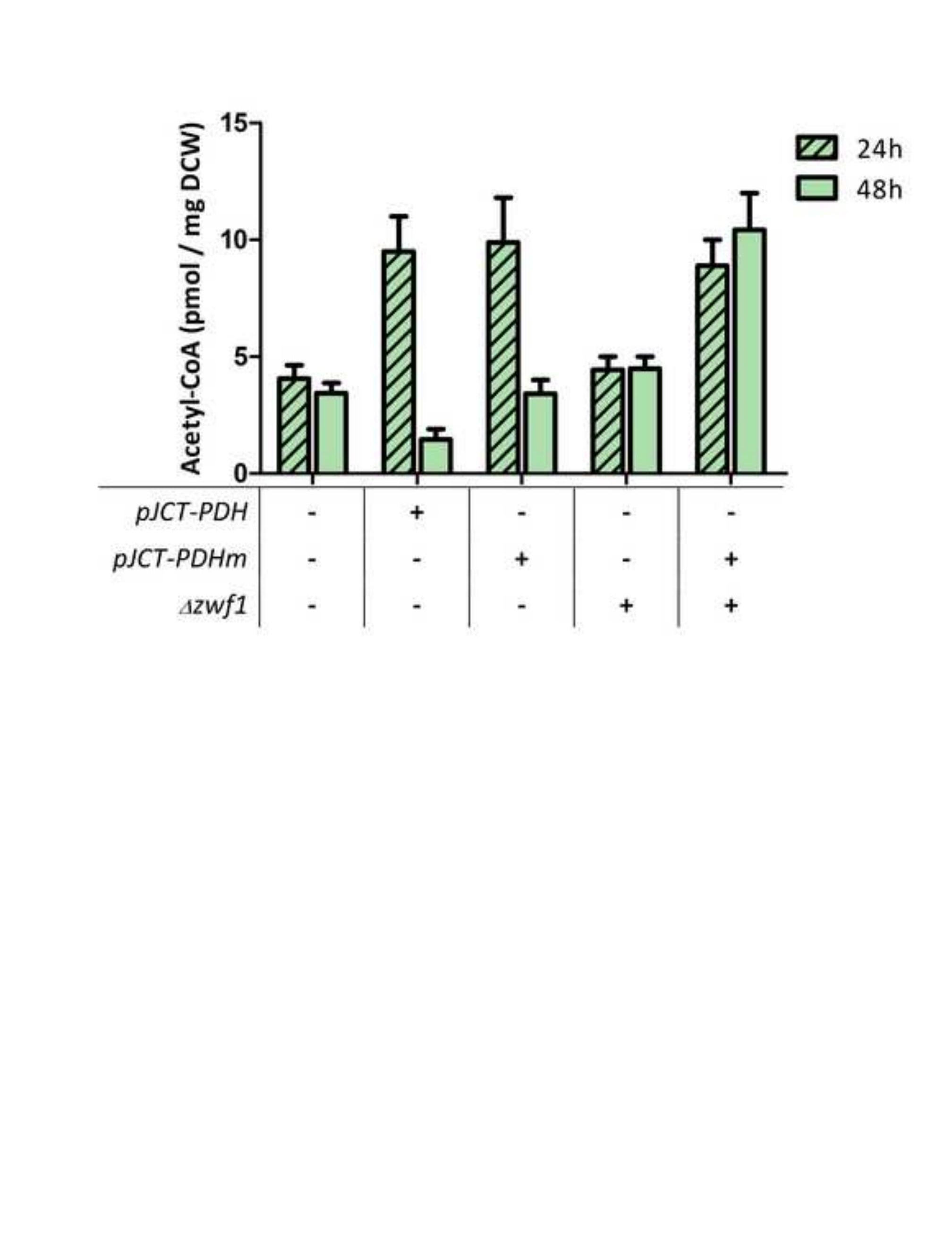
Figure 4

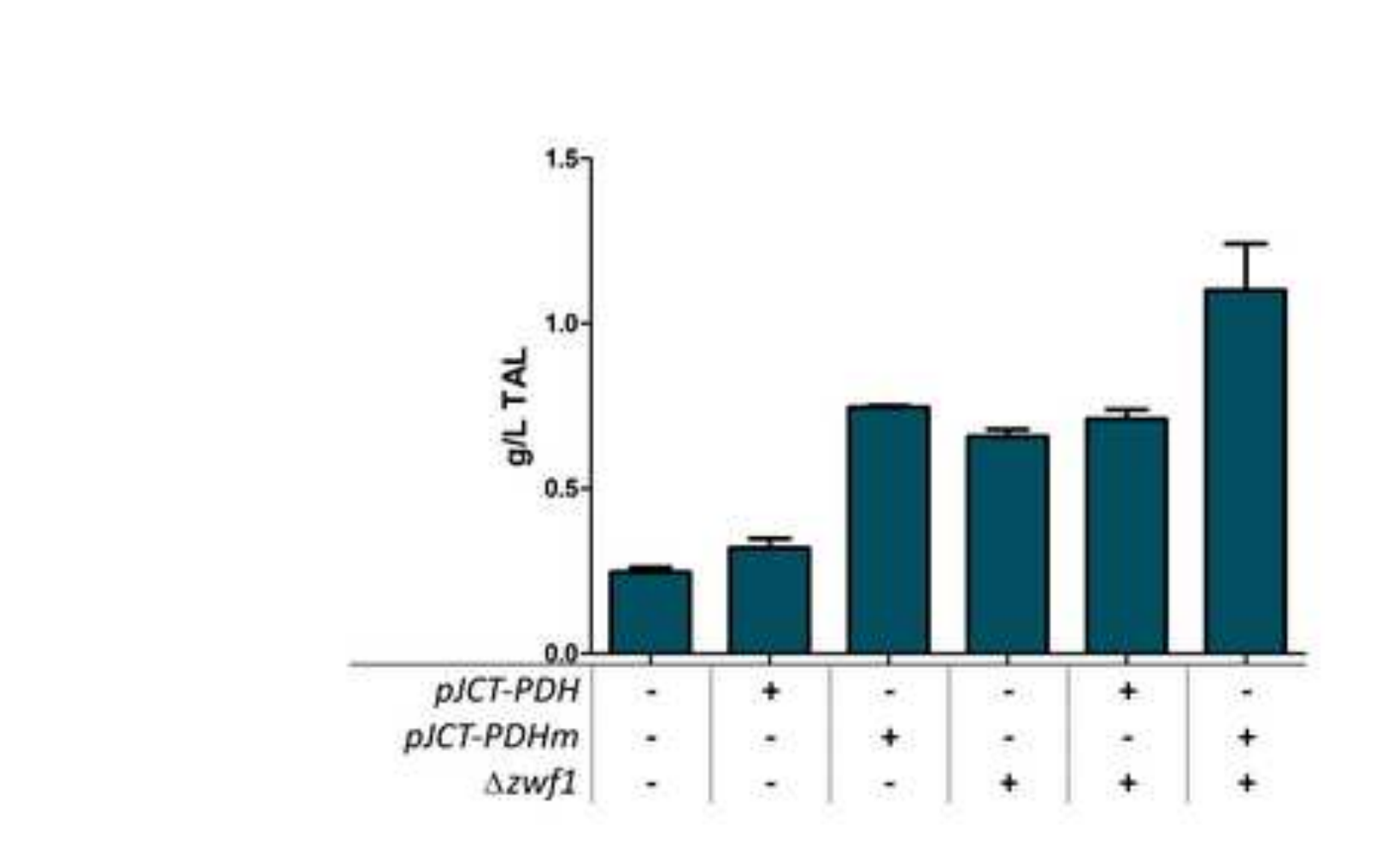

4
Figure .

(2-2

$\sqrt{2}+x^{2}$

-

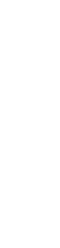

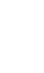

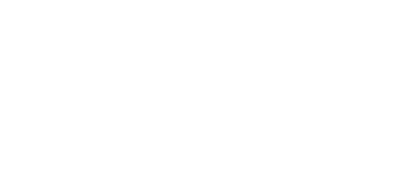

$$
\text { man }: \text { : li li: }
$$

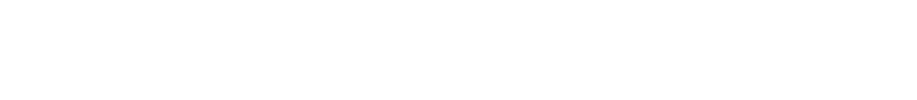




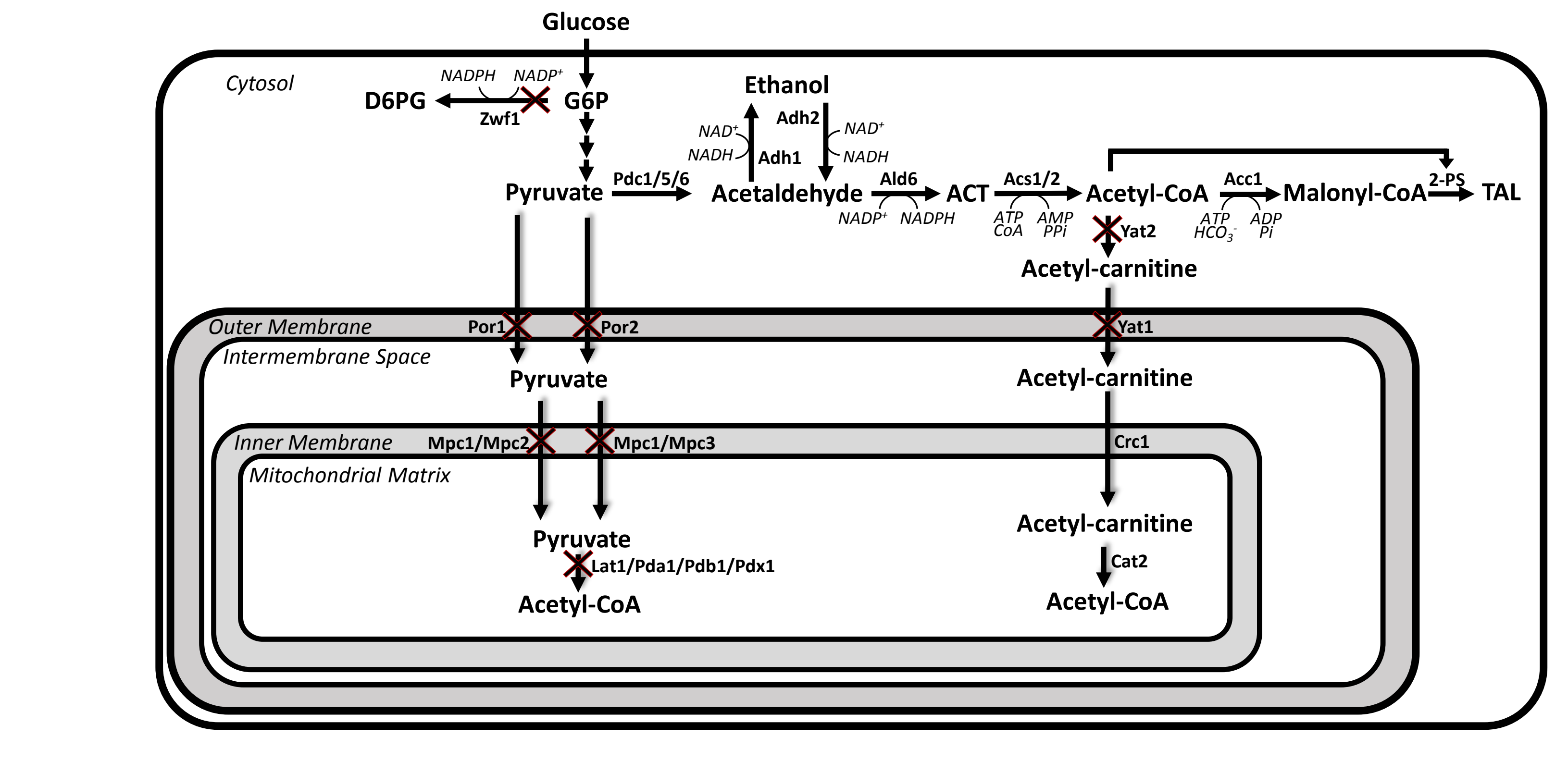


Figure 6

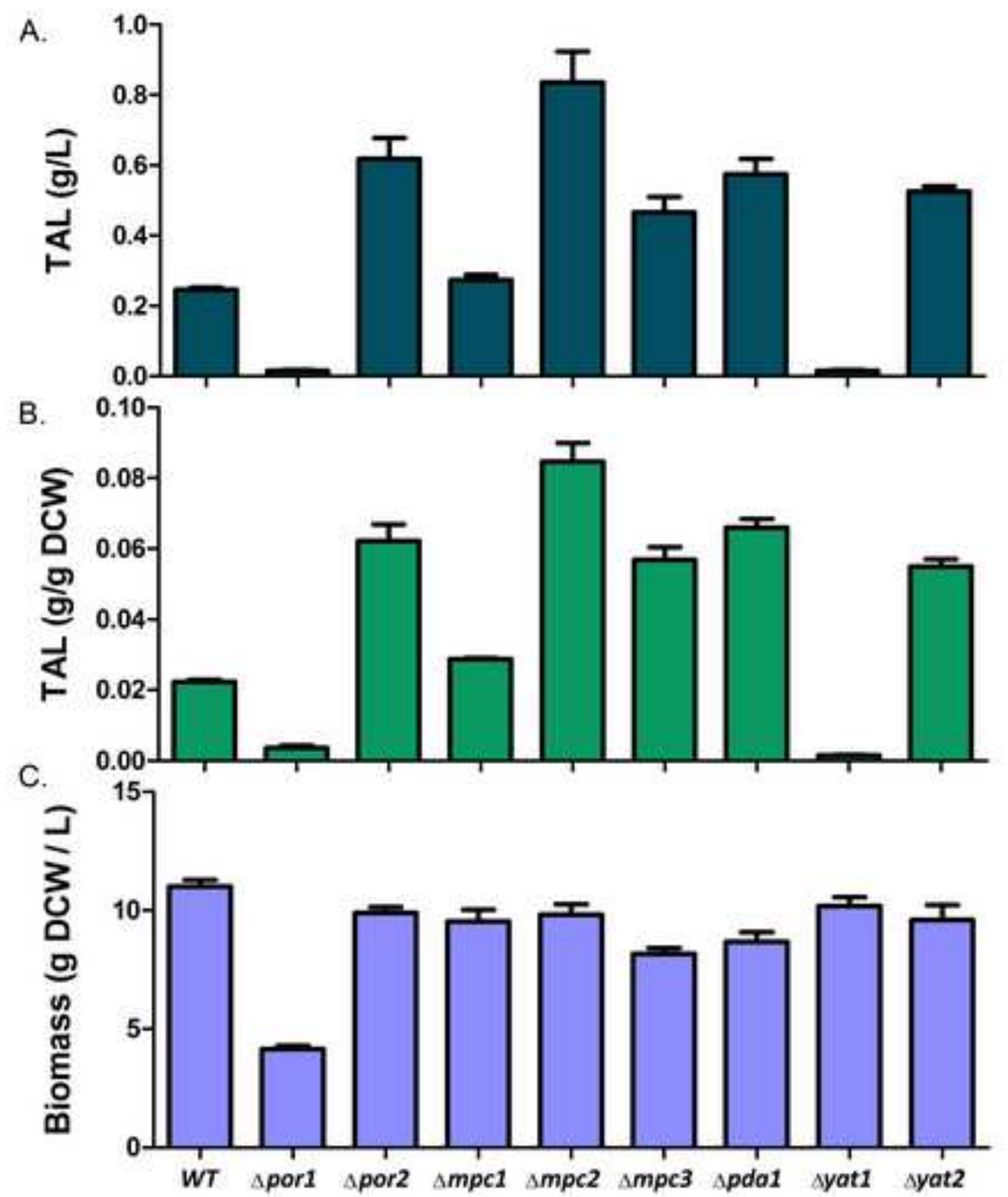




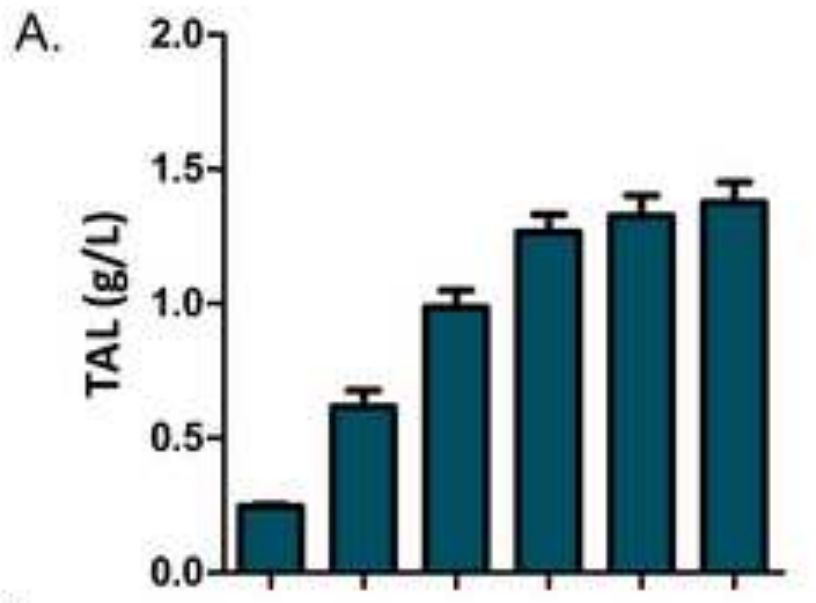

B.
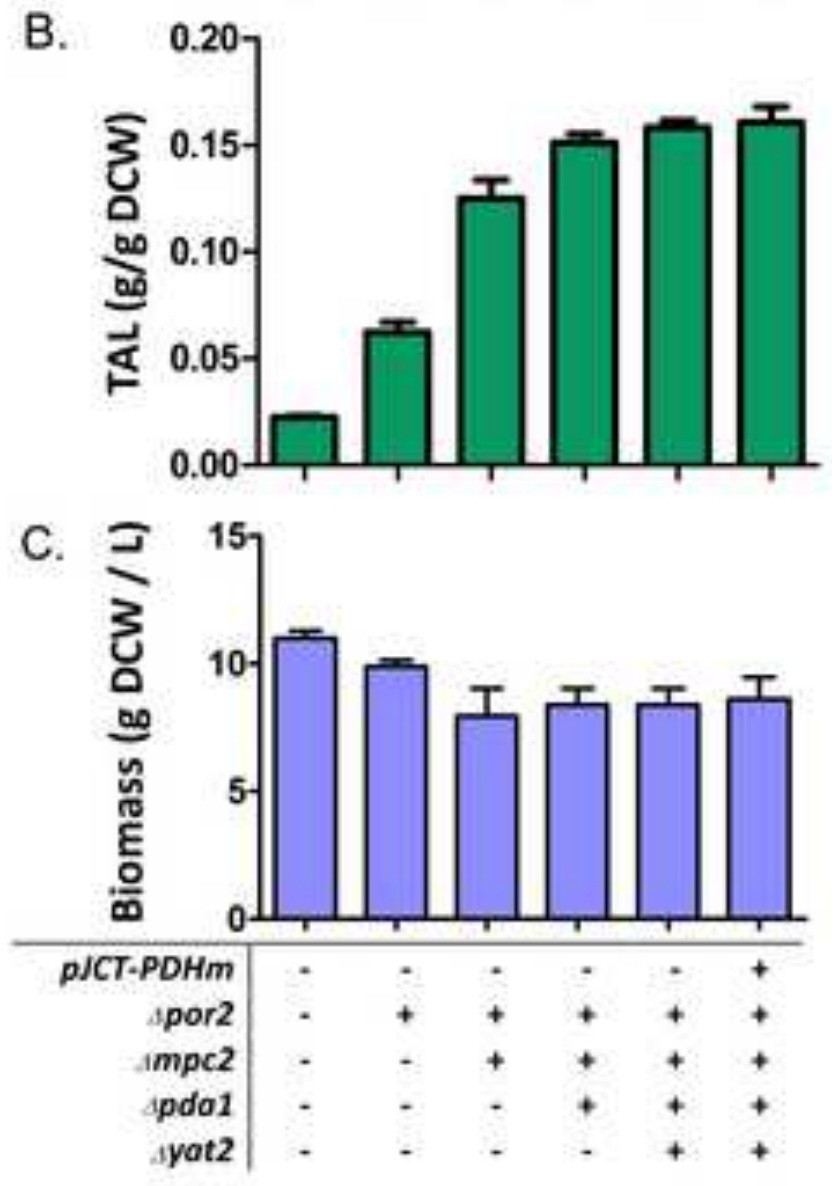
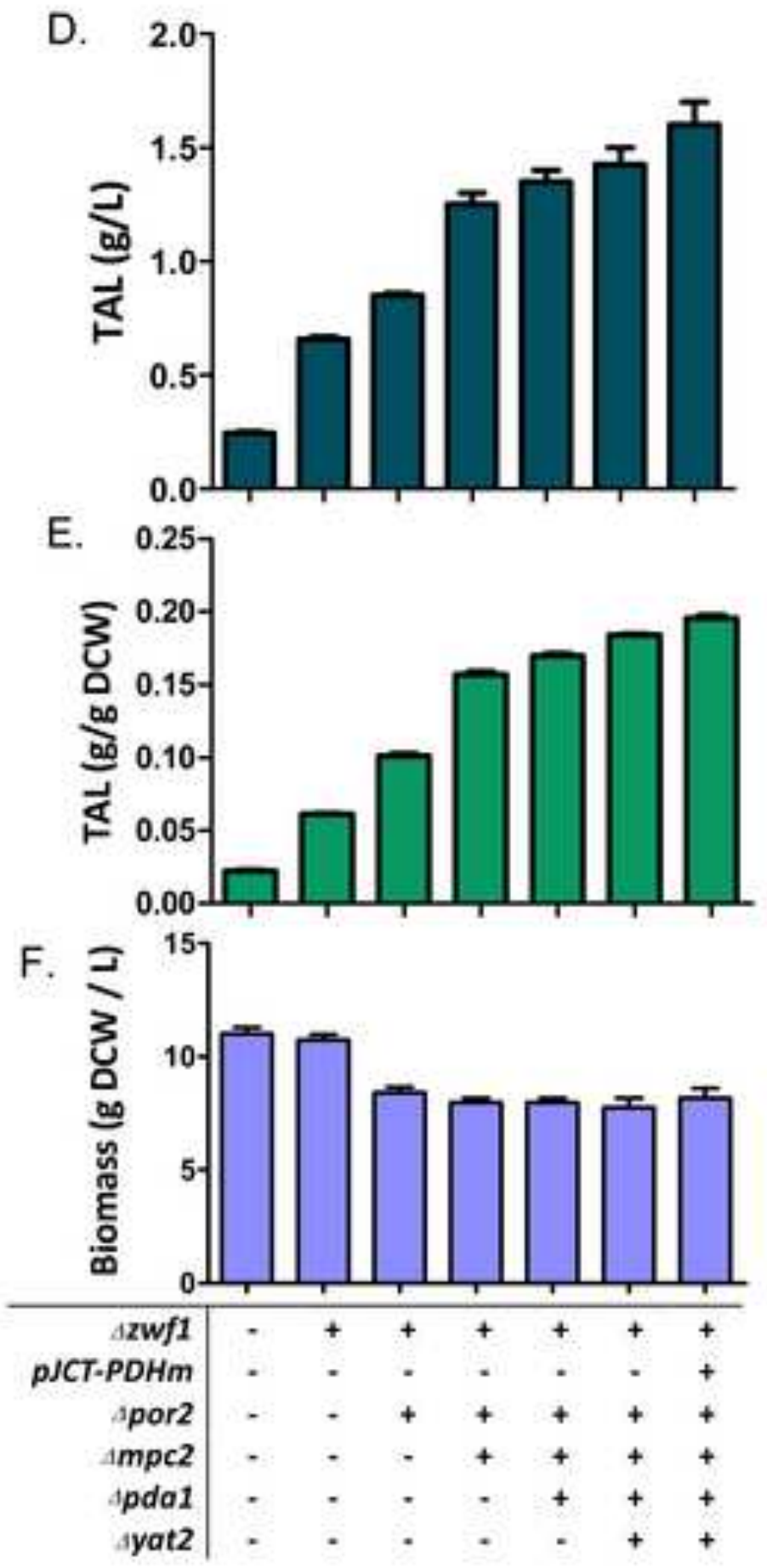


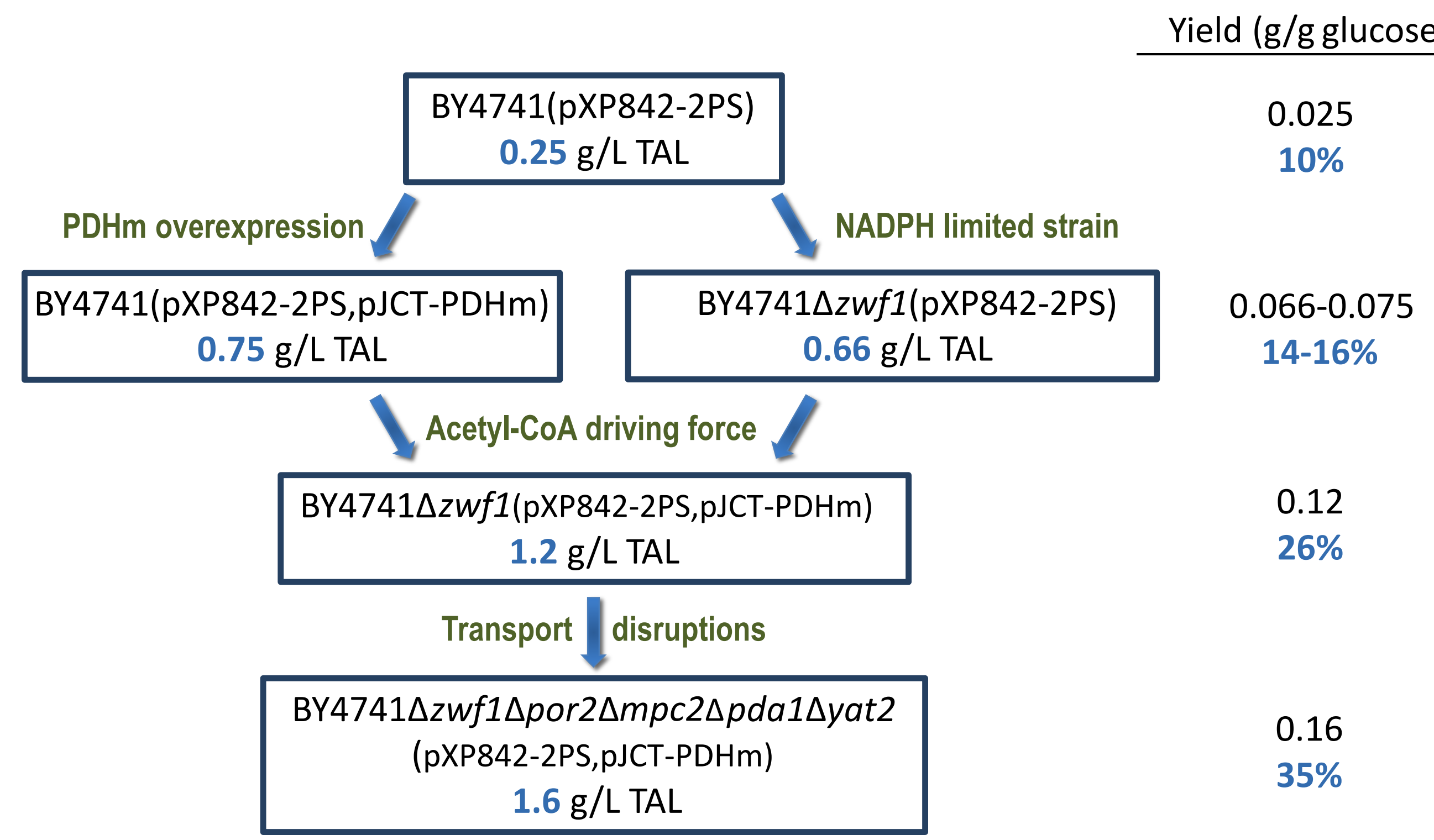

Yield (g/g glucose)

066-0.075

$14-16 \%$

0.12

$26 \%$

$35 \%$ $0.75 \mathrm{~g} / \mathrm{L}$ TAL 
Table 1. List of plasmids and strains

\begin{tabular}{|c|c|c|}
\hline Plasmids & Description & Source \\
\hline pXP842 & $2 \mu$ vector, $A D H 2$ promoter, $C Y C 1$ terminator, $U R A 3$ selectable marker & Shen et al. (2012) \\
\hline pXP843 & $2 \mu$ vector, $A D H 2$ promoter, $C Y C 1$ terminator, $T R P 1$ selectable marker & Leber and Da Silva (2014) \\
\hline pXP842-2PS & $\begin{array}{l}\text { pXP218 harboring the } g 2 p s 1 \text { gene encoding G. hybrida 2-pyrone } \\
\text { synthase }\end{array}$ & Cardenas and Da Silva (2014) \\
\hline pJCT-PDH & $\begin{array}{l}2 \mu \text { vector, } A D H 2 p \text {-lpd1-ADH2t, HXT7p-aceE-CYC1t, } \\
A D H 1 M p \text {-aceF-ADH2t, TRP1 selectable marker }\end{array}$ & This study \\
\hline pJCT-PDHm & $\begin{array}{l}2 \mu \text { vector, } A D H 2 p \text {-lpd1m-ADH2t, HXT7p-aceE-CYClt, } \\
A D H 1 M p \text {-aceF-ADH2t, TRP1 selectable marker }\end{array}$ & This study \\
\hline Strains & Description & Source \\
\hline BY4741 & MATa his $3 \Delta 1$ leu $2 \Delta 0$ met $15 \Delta 0$ ura $3 \Delta 0$ & Open Biosystems \\
\hline BY4741 $\Delta t r p 1(\mathrm{BYt})$ & BY4741 trp1::KanMX & Open Biosystems \\
\hline BY $\Delta$ por1 & BY4741 por1::KanMX & Open Biosystems \\
\hline BY $\Delta p o r 2$ & BY4741 por $2::$ KanMX & Open Biosystems \\
\hline $\mathrm{BY} \Delta m p c 1$ & BY4741 mpc1::KanMX & Open Biosystems \\
\hline $\mathrm{BY} \Delta m p c 2$ & BY4741 mpc2::KanMX & Open Biosystems \\
\hline $\mathrm{BY} \Delta m p c 3$ & BY4741 mpc3::KanMX & Open Biosystems \\
\hline $\mathrm{BY} \Delta p d a 1$ & BY4741 pda1::KanMX & Open Biosystems \\
\hline BY $\Delta y a t 1$ & BY4741 yat1::KanMX & Open Biosystems \\
\hline BYsyat2 & BY4741 yat2::KanMX & Open Biosystems \\
\hline $\mathrm{BYt} \Delta z w f 1$ & BYt $z w f 1:: M E T 15$ & This study \\
\hline $\mathrm{BYt} \Delta$ por2 & BYt por $2:: T R P 1$ & This study \\
\hline $\mathrm{BYt} \Delta$ por $2 \Delta m p c 2$ & BYt por $2:: T R P 1$ mpc2::MET15 & This study \\
\hline BYt $\Delta p o r 2 \Delta m p c 2 \Delta p d a l$ & BYt por2::TRP1 mpc2::MET15 pda1::HIS3 & This study \\
\hline $\mathrm{BYt} \Delta$ por $2 \Delta m p c 2 \Delta p d a 1 \Delta y a t 2$ & BYt por $2:: T R P 1$ mpc $2::$ MET15 pda1::HIS3 yat $2:: L E U 2$ & This study \\
\hline $\mathrm{BYtA} \Delta$ por $2 \Delta m p c 2 \Delta p d a 1 \Delta y a t 2$ & BYt por $2::$ loxP mpc $2::$ MET15 pda1::HIS3 yat $2::$ LEU2 & This study \\
\hline $\mathrm{BYt} \Delta z w f 1 \Delta$ por 2 & BYt $z w f 1:: M E T 15$ por $2:: L E U 2$ & This study \\
\hline $\mathrm{BYt} \Delta z w f 1 \Delta p o r 2 \Delta m p c 2$ & BYt zwf1::MET15 por $2:: L E U 2$ mpc $2:: H I S 3$ & This study \\
\hline $\mathrm{BYt} \Delta z w f 1 \Delta p o r 2 \Delta m p c 2 \Delta p d a 1$ & BYt zwf1::MET15 por $2::$ LEU2 mpc2::HIS3 pda1::TRP1 & This study \\
\hline BYt $\Delta z w f 1 \Delta p o r 2 \Delta m p c 2 \Delta p d a 1 \Delta y a t 2$ & BYt zwf1::MET15 por $2::$ LEU2 mpc $2: \because$ HIS 3 pda1::loxP yat $2:: T R P 1$ & This study \\
\hline $\mathrm{BYtB} \Delta z w f 1 \Delta p o r 2 \Delta m p c 2 \Delta p d a 1 \Delta y a t 2$ & BYt zwf $1::$ MET15 por $2:: L E U 2$ mpc $2:: H I S 3$ pda1::loxP yat $2:$ loxP & This study \\
\hline
\end{tabular}

